\title{
Article \\ Exploring the Relationships between Four New Species of Boletoid Fungi from Northern China and Their Related Species
}

\author{
Yang Wang ${ }^{1,2}$, Yong-Lan Tuo ${ }^{2} \oplus$, Dong-Mei $\mathrm{Wu}^{3}{ }^{3}$, Neng Gao ${ }^{3}$, Zhen-Hao Zhang ${ }^{2}$, Gu Rao ${ }^{2}$, Xiao-Min Wang ${ }^{4}$, \\ Jing Wang ${ }^{5}$, Dan Dai ${ }^{6}$, Yu Li ${ }^{2, *}$ and Bo Zhang ${ }^{2, *}$
}

1 College of Plant Protection, Shenyang Agricultural University, Shenyang 110866, China; lesireyang@163.com

2 Engineering Research Center of Chinese Ministry of Education for Edible and Medicinal Fungi, Jilin Agricultural University, Changchun 130118, China; tuoyonglan66@163.com (Y.-L.T.); zzhzz34@163.com (Z.-H.Z.); raogufungi@163.com (G.R.)

3 Biotechnology Research Institute, Xinjiang Academy of Agricultural and Reclamation Sciences, Shihezi 830011, China; wdm0999123@sina.com (D.-M.W.); gaoneng520@163.com (N.G.)

4 Institute of Soil and Fertilizer, Guizhou Academy of Agricultural Sciences, Guiyang 550006, China; rd11916@163.com

5 Institute of Biology, Guizhou Academy of Sciences, Guiyang 550001, China; 17687190411@163.com

6 Institute of Agricultural Applied Microbiology, Jiangxi Academy of Agricultural Sciences, Nanchang 330200, China; m13082447311@163.com

* Correspondence: fungi966@126.com (Y.L.); zhangbofungi@126.com (B.Z.)

\section{check for}

updates

Citation: Wang, Y.; Tuo, Y.-L.; Wu, D.-M.; Gao, N.; Zhang, Z.-H.; Rao, G.; Wang, X.-M.; Wang, J.; Dai, D.; Li, Y.; et al. Exploring the Relationships between Four New Species of Boletoid Fungi from Northern China and Their Related Species. J. Fungi 2022, 8, 218. https://doi.org/ $10.3390 /$ jof 8030218

Academic Editors: Vladimír Antonín and Hana Sevcikova

Received: 23 January 2022

Accepted: 20 February 2022

Published: 22 February 2022

Publisher's Note: MDPI stays neutral with regard to jurisdictional claims in published maps and institutional affiliations.

Copyright: (C) 2022 by the authors. Licensee MDPI, Basel, Switzerland. This article is an open access article distributed under the terms and conditions of the Creative Commons Attribution (CC BY) license (https:// creativecommons.org/licenses/by/ $4.0 /)$.

\begin{abstract}
The family Boletaceae primarily represents ectomycorrhizal fungi, which play an essential ecological role in forest ecosystems. Although the Boletaceae family has been subject to a relatively global and comprehensive history of work, novel species and genera are continually described. During this investigation in northern China, many specimens of boletoid fungi were collected. Based on the study of their morphology and phylogeny, four new species, Butyriboletus pseudoroseoflavus, Butyriboletus subregius, Tengioboletus subglutinosus, and Suillellus lacrymibasidiatus, are introduced. Morphological evidence and phylogenetic analyses of the single or combined dataset (ITS or 28S, $r p b 1, r p b 2$, and tef1) confirmed these to be four new species. The evidence and analyses indicated the new species' relationships with other species within their genera. Detailed descriptions, color photographs, and line drawings are provided. The species of Butyriboletus in China were compared in detail and the worldwide keys of Tengioboletus and Suillellus were given.
\end{abstract}

Keywords: Boletales; biodiversity; molecular analyses; taxonomy

\section{Introduction}

Boletaceae Chevall. [1], a family with more than 70 genera, is one of the most prominent and diverse among the basidiomycetes [2]. It is mainly characterized by being tubulose with infrequent lamellate or loculate hymenophora, and by a fleshy context. Most Boletaceae species have value for humans and are essential for mutualistic symbiosis with trees [3-6]. Although the family Boletaceae was established nearly two centuries ago, the species diversity of the family increased significantly in the last few decades [7-19]. Because the morphology of Boletaceae has convergent characteristics, the classification did not correspond to the phylogeny of Boletaceae for a long time. With the development of molecular biology, the method of genealogical concordance phylogenetic species recognition (GCPSR) [20] was used to identify species of fungi, resolved some doubts about the status of taxa, and contributed to a better understanding of the relationships of the genera in this family $[5,21,22]$. In the past two decades, new genera and new species have rapidly increased, and the evolution of ectomycorrhizas of Boletales was gradually disclosed $[23,24]$.

In China, the family Boletaceae has continued to receive increasing attention from mycologists [5,25-32]. However, the previous studies were focused on southern China, and 
the species diversity remained unclear in northern China. During previous field collection in the north of China, we obtained many specimens. Based on our analyses of their morphology and phylogeny, we propose four new species: Butyriboletus pseudoroseoflavus, Butyriboletus subregius, Tengioboletus subglutinosus, and Suillellus lacrymibasidiatus.

Butyriboletus was erected by Arora et al. [33] to accommodate the Boletus sect. Appendiculati. It is characterized by a reddish to brown pileus and a yellow hymenophore, usually staining blue when bruised. Five species have been described in China, i.e., Bu. huangnianlaii N.K. Zeng, H. Chai \& Zhi Q. Liang [28], Bu. pseudospeciosus Kuan Zhao \& Zhu L. Yang [5], Bu. roseoflavus (Hai B. Li \& Hai L. Wei), D. Arora \& J.L. Frank [33], Bu. sanicibus D. Arora \& J.L. Frank [33], and Bu. yicibus D. Arora \& J.L. Frank [32].

Tengioboletus was established by Wu et al. [5], including three species: T. glutinosus G. Wu \& Zhu L. Yang, T. reticulatus G. Wu \& Zhu L. Yang, and T. fujianensis N.K. Zeng \& Zhi Q. Liang [5,34]. Tengioboletus can be distinguished easily from other Boletaceae genera by combining the following characteristics: a yellow context; hymenophore that change color when injured; tubes that are concolorous with the surface; cystidia that are scattered; subfusiform-ventricose or clavate shape; and an epithelium to ixotrichodermium pileipellis.

Suillellus, typified by Boletus luridus Schaeff, was established by Murrill in 1909 [7]. According to Vizzini et al. [13], Suillellus s.str. is characterized by basidiomata that are usually slender, stipes that are cylindrical and sometimes covered with reticulation, pileus that are reddish brown to olivaceous and turn to blue when bruised, the presence or absence of Bataille's line, and a context that is reddish in the stipe base and bluing when exposed to air and positive amyloid reaction.

\section{Materials and Methods}

\subsection{Samplings and Morphological Analyses}

Materials were collected from Jilin province and the Xinjiang Uygur Autonomous Region, China. Voucher specimens were deposited in the Herbarium of Mycology of the Jilin Agriculture University (HMJAU). Descriptions of the colors of basidiomata used color coding from Kornerup and Wanscher [35]. The micro-morphological structures were performed in a $5 \% \mathrm{KOH}$ solution and then in a 1\% Congo Red or Melzer's reagent solution. The amyloid reaction was tested following Imler's procedure [36,37]. The abbreviations for basidiospore measurements $(\mathrm{n} / \mathrm{m} / \mathrm{p}$ ) indicate " $\mathrm{n}$ " basidiospores from " $\mathrm{m}$ " basidiomata of " $p$ " specimens. The sizes of basidiospores are given as (a) b-m-c (d), where "a" is the smallest value, " $\mathrm{d}$ " is the largest value, " $\mathrm{m}$ " is the average value point, and " $\mathrm{b}-\mathrm{c}$ " covers a minimum of $95 \%$ of the values. "Q" stands for the ratio of the length and the width of the basidiospores and " $\mathrm{Q} \pm \mathrm{av}$ " stands for for the average $\mathrm{Q}$ of all basidiospores \pm sample standard deviation. The scanning electron microscope (SEM) was used to observe the ultrastructure of the spores.

\subsection{DNA Extraction, PCR Amplification, and Sequencing}

Genomic DNA was extracted from dried specimens, using the NuClean Plant Genomic DNA kit (CWBIO). For the amplification of ITS, 28S, $r p b 1, r p b 2$, and tef1, we used primer pairs ITS1/4, LROR/LR5, RPB1-B-F/RPB1-B-R, RPB2-B-F1/RPB2-B-R, and 983F/1567R, respectively $[5,22,25,38-42]$. PCR amplification procedures were set to refer to Zhang et al. [43], White et al. [39], and Kuo and Ortiz-Santana [44]. Then, PCR productions were sent to Sangon Biotech Co. Ltd. (Shanghai, China) to be directly sequenced using the ABI 3730xl DNA analyzer.

\subsection{Data Analysis}

Newly generated sequences were uploaded to NCBI (https:/ / www.ncbi.nlm.nih.gov/, accessed on 10 January 2022), as shown in Table 1, with other similar sequences downloaded from the NCBI and UNITE (https:/ / unite.ut.ee/, accessed on 10 January 2022) datasets. DNA sequences were aligned and manually modified using Bioedit v7.1.3 [45]. In the multi-locus dataset $(28 \mathrm{~S}+r p b 1+r p b 2+t e f 1)$ of Tengioboletus, $894 \mathrm{bp}$ for 28S, $758 \mathrm{bp}$ for $r p b 1$, 
$710 \mathrm{bp}$ for $r p b 2$, and $638 \mathrm{bp}$ for tef 1 , and in the four-locus dataset $(t e f 1+28 \mathrm{~S}+r p b 2+$ ITS) of Butyriboletus, $730 \mathrm{bp}$ for tef $1,863 \mathrm{bp}$ for $28 \mathrm{~S}, 834 \mathrm{bp}$ for $r p b 2$, and $809 \mathrm{bp}$ for ITS. The data used for phylogenetic analyses for Suillellus included the ITS dataset and a multi-locus dataset $(t e f 1+28 \mathrm{~S}+r p b 1+r p b 2)$, For the multi-locus dataset, $907 \mathrm{bp}$ for 28S, $791 \mathrm{bp}$ for $r p b 1,719 \mathrm{bp}$ for $r p b 2$, and $631 \mathrm{bp}$ for tef1. The best models of the multi-locus datasets were searched via PartitionFinder 2 [46]. Meanwhile, the best model of the ITS dataset was searched via Modelfinder [47]. Phylogenetic analyses were carried out using the maximum likelihood method (ML) and the Bayes inference (BI) method. The models employed for each of the four loci of Tengioboletus, and Butyriboletus were GTR + I + G for 28S, SYM + G for $r p b 1, \mathrm{~K} 80$ + G for $r p b 2, \mathrm{SYM}+\mathrm{I}+\mathrm{G}$ for tef1, and GTR + I + G for ITS, GTR + I + G for 28S, $\mathrm{K} 80+\mathrm{G}$ for $r p b 2, \mathrm{SYM}+\mathrm{I}+\mathrm{G}$ for tef1, respectively. For the multi-locus dataset of Suillellus, the best models for each locus were K80 + I + G for $r p b 1$ and SYM + I + G for 28S, $r p b 2$, and tef1. In the ITS dataset of Suillellus, the best models for ML analysis and BI analyses were K2P + I + G4. For ML analyses, the datasets were analyzed using IQ-TREE [48] under an ultrafast bootstrap, with 5000 replicates. For BI analyses, the multi-locus datasets were analyzed using MrBayes 3.2.6 [49], running in a total of 2,000,000 generations, and sampled every 1000 generations. The initial $25 \%$ of the sampled data were discarded as burn-in. Other parameters were kept at their default settings.

Table 1. Information of DNA sequences used to reconstruct phylogenetic trees. Sequences newly generated in this study are indicated in bold.

\begin{tabular}{|c|c|c|c|c|c|c|c|}
\hline Taxon & Voucher ID & ITS & $28 S$ & TEF1 & RPB1 & RPB2 & References \\
\hline Tengioboletus glutinosus & HKAS53425 & - & KF112341 & KF112204 & KF112578 & KF112800 & [22] \\
\hline T. glutinosus & HKAS53452 & - & KT990655 & KT990844 & KT990994 & KT990480 & [5] \\
\hline T. reticulatus & HKAS53426 & - & KF112491 & KF112313 & KF112649 & KF112828 & [22] \\
\hline T. reticulatus & HKAS52241 & - & KT990657 & KT990845 & KT990995 & KT990481 & [5] \\
\hline T. reticulatus & HKAS53453 & - & KT990656 & KT990846 & - & KT990482 & [5] \\
\hline T. funjianensis & HKAS76661 & - & KF112342 & KF112205 & - & KF112801 & [22] \\
\hline T. funjianensis & HKAS77869 & - & KT990658 & KT990847 & KT990996 & KT990483 & [5] \\
\hline T. subglutinosus & $\begin{array}{l}\text { HMJAU59034 } \\
\text { (T286) }\end{array}$ & - & OL588198 & OL739119 & OL739121 & - & this study \\
\hline T. subglutinosus & $\begin{array}{l}\text { HMJAU59035 } \\
\text { (T293) }\end{array}$ & - & OL588197 & OL739120 & OL739122 & OL739118 & this study \\
\hline $\begin{array}{l}\text { Porphyrellus } \\
\text { porphyrosporus }\end{array}$ & MB97-023 & - & DQ534643 & GU187734 & GU187475 & GU187800 & {$[50]$} \\
\hline P. porphyrosporus & HKAS76671 & - & KF112482 & KF112243 & KF112611 & KF112718 & [22] \\
\hline Tylopilus sp. & HKAS50211 & - & KT990552 & KT990752 & KT990920 & KT990389 & [22] \\
\hline Tylopilus sp. & HKAS59826 & - & KT990558 & - & - & - & [5] \\
\hline Tylopilus sp. & HKAS90198 & - & KT990559 & - & - & - & [5] \\
\hline $\begin{array}{l}\text { Strobilomyces } \\
\text { atrosquamosus }\end{array}$ & HKAS55368 & - & KT990648 & KT990839 & KT990989 & KT990476 & [5] \\
\hline S. atrosquamosus & HKAS78563 & - & KT990649 & KT990833 & КT990983 & KT990470 & [5] \\
\hline S. echinocephalus & HKAS59420 & - & KF112463 & KF112256 & KF112600 & KF112810 & [22] \\
\hline P. aff. alboater & HKAS55375 & - & KT990622 & KT990816 & КТ990969 & - & [5] \\
\hline P. nigropurpureus & HKAS74938 & - & KF112466 & KF112246 & - & KF112763 & [22] \\
\hline P. nigropurpureus & HKAS52685 & - & KT990627 & KT990821 & KT990973 & KT990459 & [5] \\
\hline P. nigropurpureus & HKAS53370 & - & KT990628 & KT990822 & KT990974 & KT990460 & [5] \\
\hline P. holophaeus & HKAS50508 & - & KF112465 & KF112244 & KF112553 & - & [22] \\
\hline P. holophaeus & HKAS74894 & - & KF112474 & KF112245 & KF112554 & - & [22] \\
\hline P. castaneus & HKAS63076 & - & KT990548 & KT990749 & KT990916 & KT990386 & [5] \\
\hline P. castaneus & HKAS52554 & - & KT990697 & KT990883 & KT991026 & KT990502 & [5] \\
\hline P. orientifumosipes & HKAS75078 & - & KF112481 & KF112242 & - & KF112717 & [22] \\
\hline P. orientifumosipes & HKAS53372 & - & KT990629 & KT990823 & KT990975 & KT990461 & [5] \\
\hline Boletus bainiugan & HKAS52235 & - & KF112457 & KF112203 & KF112587 & KF112705 & [22] \\
\hline B. bainiugan & HKAS55393 & - & JN563852 & - & JN563868 & - & [51] \\
\hline B. fagacicola & HKAS55975 & - & JN563853 & - & JN563879 & - & [51] \\
\hline B. fagacicola & HKAS71347 & - & JQ172790 & - & JQ172791 & - & [51] \\
\hline Xanthoconium affine & $\begin{array}{l}\text { NY00815399 } \\
\text { (REH8660) }\end{array}$ & - & KT990661 & KT990850 & KT990999 & KT990486 & [5] \\
\hline X. porophyllum & HKAS90217 & - & KT990662 & KT990851 & KT991000 & KT990487 & [5] \\
\hline Baorangia pseudocalopus & HKAS63607 & - & KF112355 & KF112167 & - & - & [22] \\
\hline Ba. pseudocalopus & HKAS75081 & - & KF112356 & KF112168 & - & - & [22] \\
\hline Butyriboletus abieticola & Arora11087 & KC184412 & KC184413 & - & - & - & [33] \\
\hline Bu. appendiculatus & Bap1 & KJ419923 & AF456837 & JQ327025 & - & - & [52] \\
\hline Bu. appendiculatus & BR50200893390-25 & KT002598 & KT002609 & КT002633 & - & - & [53] \\
\hline Bu. appendiculatus & BR50200892955-50 & KJ605668 & KJ605677 & KJ619472 & - & KР055030 & [54] \\
\hline Bu. appendiculatus & MB000286 & KT002599 & KT002610 & KT002634 & - & - & [53] \\
\hline
\end{tabular}


Table 1. Cont.

\begin{tabular}{|c|c|c|c|c|c|c|c|}
\hline Taxon & Voucher ID & ITS & $28 S$ & TEF1 & RPB1 & RPB2 & References \\
\hline Bu. autumniregius & Arora11108 & KC184423 & KC184424 & - & - & - & [33] \\
\hline Bu. brunneus & NY00013631 & KT002600 & KT002611 & KT002635 & - & - & [53] \\
\hline Bu. fechtneri & AT2003097 & KC584784 & KF030270 & - & - & - & [21] \\
\hline Exsudoporus frostii & JLF2548 & KC812303 & KC812304 & - & - & - & [33] \\
\hline E. frostii & NY815462 & - & JQ924342 & KF112164 & - & KF112675 & [22] \\
\hline E. floridanus & BOS 617, BZ 3170 & MN250222 & MK601725 & MK721079 & - & MK766287 & [43] \\
\hline Bu. hainanensis & $\begin{array}{l}\text { N.K. Zeng } 1197 \\
\text { (FHMU 2410) }\end{array}$ & KU961653 & KU961651 & - & - & KU961658 & {$[32]$} \\
\hline Bu. hainanensis & $\begin{array}{l}\text { N.K. Zeng 2418 } \\
\text { (FHMU 2437) }\end{array}$ & KU961654 & KU961652 & KU961656 & - & KX453856 & {$[32]$} \\
\hline Bu. huangnianlaii & $\begin{array}{l}\text { N.K. Zeng } 3245 \\
\text { (FHMU 2206) }\end{array}$ & MH885350 & MH879688 & MH879717 & - & MH879740 & [28] \\
\hline Bu. huangnianlaii & $\begin{array}{l}\text { N.K. Zeng } 3246 \\
\text { (FHMU 2207) }\end{array}$ & MH885351 & MH879689 & MH879718 & - & MH879741 & [28] \\
\hline Bu. peckii & 3959 & - & JQ326999 & JQ327026 & - & - & [55] \\
\hline Bu. persolidus & Arora11110 & KC184444 & - & - & - & - & [33] \\
\hline Bu. primiregius & DBB00606 & - & KC184451 & - & - & - & [33] \\
\hline Bu. fuscoroseus & BR50201618465-02 & KT002602 & KT002613 & KT002637 & - & - & [53] \\
\hline Bu. fuscoroseus & BR50201533559-51 & KT002603 & KT002614 & KT002638 & - & - & [53] \\
\hline Bu. pseudospeciosus & HKAS59467 & - & KF112331 & KF112176 & - & KF112672 & [22] \\
\hline Bu. pseudospeciosus & HKAS63513 & - & KT990541 & KT990743 & - & KT990380 & [5] \\
\hline Bu. pseudospeciosus & HKAS63596 & - & KT990542 & KT990744 & - & KT990381 & [5] \\
\hline Bu. pseudospeciosus & $\begin{array}{l}\text { N.K. Zeng } 2127 \\
\text { (FHMU 1391) }\end{array}$ & MH885349 & MH879687 & MH879716 & - & - & [28] \\
\hline Bu. fuscoroseus & MG383a & KC184458 & - & - & - & - & [33] \\
\hline Bu. pulchriceps & DS4514 & - & KF030261 & KF030409 & - & - & [21] \\
\hline Bu. pulchriceps & R. Chapman 0945 & KT002604 & KT002615 & KT002639 & - & - & [53] \\
\hline Bu. querciregius & Arora11100 & KC184461 & - & - & - & - & [33] \\
\hline Bu. regius & MB000287 & KT002605 & KT002616 & КТ002640 & - & - & [53] \\
\hline Bu. regius & MG408a & KC584789 & KC584790 & - & - & - & [33] \\
\hline Bu. regius & PRM:923465 & KJ419920 & KJ419931 & - & - & - & [56] \\
\hline Bu. roseoflavus & Arora11054 & KC184434 & KC184435 & - & - & - & [33] \\
\hline Bu. roseoflavus & HKAS63593 & KJ909517 & KJ184559 & KJ184571 & - & - & [53] \\
\hline Bu. roseoflavus & HKAS54099 & KJ909519 & KF739665 & KF739779 & - & - & [53] \\
\hline Bu. roseoflavus & $\begin{array}{l}\text { N.K. Zeng } 2123 \\
\text { (FHMU 1387) }\end{array}$ & MH885348 & MH879686 & MH879715 & - & - & [28] \\
\hline Bu. pseudoroseoflavus & $\begin{array}{l}\text { HMJAU59470 } \\
\text { (T274) }\end{array}$ & OL604164 & OL587853 & OL739124 & - & OL739126 & this study \\
\hline Bu. pseudoroseoflavus & $\begin{array}{c}\text { HMJAU59471 } \\
\text { (R383) }\end{array}$ & OL604165 & OL587852 & OL739123 & - & OL739125 & this study \\
\hline Bu. Roseopurpureus & E.E. Both3765 & KT002606 & КТ002617 & KT002641 & - & - & [53] \\
\hline Bu. Roseopurpureus & JLF2566 & KC184466 & KC184467 & - & - & - & [33] \\
\hline Bu. Roseopurpureus & MB06-059 & KC184464 & KF030262 & KF030410 & - & - & [21] \\
\hline Bu. sanicibus & Arora99211 & KC184469 & KC184470 & - & - & - & [33] \\
\hline Bu. subregius & $\begin{array}{l}\text { HMJAU60200 } \\
\text { (T95) }\end{array}$ & OM237336 & OM237339 & OM285111 & - & OM285109 & this study \\
\hline Bu. subregius & $\begin{array}{l}\text { HMJAU60201 } \\
\text { (T198) }\end{array}$ & OM237337 & OM237340 & OM285112 & - & OM285110 & this study \\
\hline Butyriboletus sp. & MHHNU7456 & - & КТ990539 & КT990741 & - & KT990378 & [5] \\
\hline Butyriboletus sp. & HKAS52525 & - & KF112337 & KF112163 & - & KF112671 & [22] \\
\hline Butyriboletus sp. & HKAS57774 & - & KF112330 & KF112155 & - & KF112670 & [22] \\
\hline Bu. hainanensis & HKAS59814 & - & KF112336 & KF112199 & - & KF112699 & [22] \\
\hline Butyriboletus yicibus & HKAS63528 & - & KF112332 & KF112156 & - & KF112673 & [22] \\
\hline Bu. Subappendiculatus & MB000260 & KT002607 & KT002618 & KT002642 & - & - & [53] \\
\hline Bu. subsplendidus . & HKAS52661 & - & KF112339 & KF112169 & - & KF112676 & {$[5]$} \\
\hline Bu. taughannockensis & 263101 & MH257559 & MH236172 & - & - & - & \\
\hline Bu. taughannockensis & 250839 & MH234472 & MH234473 & - & - & - & \\
\hline Bu. taughannockensis & 252208 & MH236100 & MH236099 & - & - & - & \\
\hline Bu. yicibus & Arora9727 & KC184474 & KC184475 & - & - & - & [33] \\
\hline Bu. yicibus & HKAS57503 & KT002608 & KT002620 & KT002644 & - & - & [53] \\
\hline Bu. yicibus & HKAS68010 & KJ909521 & KT002619 & KT002643 & - & - & [53] \\
\hline Gymnogaster boletoides & $\begin{array}{l}\text { NY01194009 } \\
\text { (REH9455) }\end{array}$ & - & KT990572 & KT990768 & - & KT990406 & {$[5]$} \\
\hline $\begin{array}{l}\text { Rugiboletus } \\
\text { brunneiporus }\end{array}$ & HKAS83209 & - & KM605134 & KM605144 & - & KM605168 & [27] \\
\hline R. extremiorientalis & HKAS63635 & - & KF112403 & KF112198 & - & KF112720 & [22] \\
\hline $\begin{array}{l}\text { Crocinoboletus } \\
\text { laetissimus }\end{array}$ & HKAS50232 & - & KT990567 & KT990762 & KT990925 & - & [5] \\
\hline C. laetissimus & HKAS59701 & - & KF112436 & - & - & KF112711 & [22] \\
\hline C. rufoaureus & HKAS53424 & - & KF112435 & KF112206 & KF112533 & KF112710 & [22] \\
\hline C. rufoaureus & HKAS59820 & - & KF112434 & - & KF112532 & KF112709 & [22] \\
\hline $\begin{array}{l}\text { Cyanoboletus } \\
\text { brunneoruber }\end{array}$ & HKAS63504 & - & KF112368 & KF112194 & KF112531 & KF112702 & [22] \\
\hline Cy. brunneoruber & HKAS80579 (1) & - & KT990568 & КТ990763 & KT990926 & КT990401 & [5] \\
\hline Cy. brunneoruber & HKAS80579 (2) & - & KT990569 & KT990764 & KT990927 & KT990402 & [5] \\
\hline Cy. instabilis & HKAS59554 & - & KF112412 & KF112186 & KF112528 & KF112698 & [22] \\
\hline Cy. pulverulentus & 9606 & - & KF030313 & KF030418 & KF030364 & - & [21] \\
\hline
\end{tabular}


Table 1. Cont.

\begin{tabular}{|c|c|c|c|c|c|c|c|}
\hline Taxon & Voucher ID & ITS & $28 S$ & TEF1 & RPB1 & RPB2 & References \\
\hline Baorangia pseudocalopus & HKAS63607 & - & KF112355 & KF112167 & KF112519 & KF112677 & [22] \\
\hline Ba. pseudocalopus & HKAS75081 & - & KF112356 & KF112168 & KF112520 & KF112678 & [22] \\
\hline Lanmao angustispora & HKAS74765 & - & KF112322 & KF112159 & KF112521 & KF112680 & {$[22]$} \\
\hline L. angustispora & HKAS74752 & - & KM605139 & KM605154 & KM605166 & KM605177 & [27] \\
\hline L. angustispora & HKAS74759 & - & KM605140 & KM605155 & KM605167 & KM605178 & [27] \\
\hline L. asiatica & HKAS54094 & - & KF112353 & KF112161 & KF112522 & KF112682 & [22] \\
\hline L. asiatica & HKAS63516 & - & KT990584 & KT990780 & KT990935 & KT990419 & [5] \\
\hline L. fragrans & 18555 & JF907800 & - & - & - & - & \\
\hline $\begin{array}{l}\text { Neoboletus } \\
\text { brunneissimus }\end{array}$ & HKAS52660 & - & KF112314 & KF112143 & KF112492 & KF112650 & [22] \\
\hline N. hainanaensis & HKAS63515 & - & KT990614 & KT990808 & KT990964 & КT990449 & [5] \\
\hline N. ferrugineus & HKAS77617 & - & KT990595 & KT990788 & KT990943 & KT990430 & [5] \\
\hline N. ferrugineus & HKAS77718 & - & KT990596 & KT990789 & KT990944 & KT990431 & {$[5]$} \\
\hline N. flavidus & HKAS59443 & - & KU974139 & KU974136 & KU974142 & KU974144 & [5] \\
\hline N. flavidus & HKAS58724 & - & KU974140 & KU974137 & KU974143 & KU974145 & [5] \\
\hline Porphyrellus castaneus & HKAS52554 & - & KT990697 & KT990883 & KT991026 & KT990502 & {$[5]$} \\
\hline $\begin{array}{l}\text { P. castaneus } \\
\text { Sulu }\end{array}$ & HKAS63076 & - & KT990548 & KT990749 & KT990916 & KT990386 & {$[5]$} \\
\hline P. castaneus & HKAS68575 & - & KT990560 & - & - & - & [5] \\
\hline P. holophaeus & HKAS59407 & - & KT990708 & KT990888 & KT991030 & КT990506 & [5] \\
\hline P. nigropurpureus & HKAS52685 & - & KT990627 & KT990821 & KT990973 & KT990459 & [5] \\
\hline P. nigropurpureus & HKAS53370 & - & KT990628 & KT990822 & KT990974 & KT990460 & {$[5]$} \\
\hline P. orientifumosipes & HKAS75078 & - & KF112481 & KF112242 & - & KF112717 & [22] \\
\hline P. orientifumosipes & HKAS53372 & - & KT990629 & KT990823 & KT990975 & KT990461 & [5] \\
\hline Rubroboletus dupainii & JAM0607 & - & - & KF030413 & KF030361 & - & [21] \\
\hline R. latisporus & HKAS63517 & - & КР055022 & КР055019 & KP055025 & КР055028 & [25] \\
\hline R. latisporus & HKAS80358 & - & КР055023 & KP055020 & KP055026 & КР055029 & [25] \\
\hline R. rhodosanguineus & 4252 & - & KF030252 & KF030412 & - & - & [21] \\
\hline R. rhodoxanthus & HKAS84879 & - & KT990637 & KT990831 & KT990981 & KT990468 & [5] \\
\hline R. sinicus & HKAS68620 & - & KF112319 & KF112146 & KF112504 & KF112661 & [22] \\
\hline R. sinicus & HKAS56304 & - & KJ605673 & KJ619483 & KJ619482 & КР055031 & [54] \\
\hline Suillellus amygdalinus & $112605 \mathrm{ba}$ & - & JQ326996 & JQ327024 & KF030360 & - & [55] \\
\hline S. amygdalinus & $\begin{array}{l}\text { NY00035656 } \\
\text { (Thiers54483) }\end{array}$ & - & KT990650 & КТ990840 & KT990990 & KT990477 & [5] \\
\hline S. subamygdalinus & $\begin{array}{c}\text { (Thiers54483) } \\
\text { HKAS57262 }\end{array}$ & - & KF112316 & KF112174 & KF112501 & KF112660 & [22] \\
\hline S. subamygdalinus & HKAS53641 & - & KT990651 & KT990841 & KT990991 & KT990478 & [5] \\
\hline S. subamygdalinus & HKAS57953 & - & KT990652 & KT990842 & KT990992 & - & {$[5]$} \\
\hline S. subamygdalinus & HKAS74745 & - & KT990653 & KT990843 & KT990993 & КТ990479 & [5] \\
\hline S. lacrymibasidiatus & $\begin{array}{l}\text { HMJAU60202 } \\
\text { (W3194) }\end{array}$ & OM237315 & OM230174 & OM285117 & OM285113 & OM285115 & this study \\
\hline S. lacrymibasidiatus & $\begin{array}{c}\text { HMJAU60203 } \\
\text { (W3229) }\end{array}$ & OM237338 & OM230172 & OM285116 & - & OM285114 & this study \\
\hline Sutorius eximius & REH9400 & - & JQ327004 & JQ327029 & - & - & [55] \\
\hline Su. eximius & HKAS52672 & - & KF112399 & KF112207 & KF112584 & KF112802 & [22] \\
\hline Su. luridiformis & AT1998054 & UDB000658 & - & - & - & - & \\
\hline Tylopilus alpinus & HKAS55438 & - & KF112404 & KF112191 & KF112538 & KF112687 & [22] \\
\hline Ty. argillaceus & HKAS90201 & - & KT990588 & KT990783 & - & - & [5] \\
\hline Ty. argillaceus & HKAS90186 & - & KT990589 & KT990784 & - & KT990424 & [5] \\
\hline Ty. atripurpureus & HKAS50208 & - & KF112472 & KF112283 & KF112620 & KF112799 & [22] \\
\hline Ty. badiceps & MB03-052 & - & KF030336 & - & - & - & {$[21]$} \\
\hline Ty. badiceps & 78206 & - & KF030335 & KF030429 & - & - & [21] \\
\hline Veloporphyrellus alpinus & HKAS68301 & - & JX984538 & JX984550 & - & - & {$[57]$} \\
\hline V.alpinus & HKAS57490 & - & KF112380 & KF112209 & KF112555 & KF112733 & [22] \\
\hline V.conicus & BZ2408 & - & JX984545 & - & - & - & [57] \\
\hline V. conicus & BZ1670 & - & JX984543 & JX984555 & - & - & [57] \\
\hline V. gracilioides & HKAS53590 & - & KF112381 & KF112210 & KF112556 & KF112734 & [22] \\
\hline Zangia citrina & HKAS52677 & - & HQ326940 & HQ32687 & - & - & [58] \\
\hline Z. citrina & HKAS52684 & - & HQ326941 & HQ326872 & - & - & [58] \\
\hline Z. erythrocephala & HKAS52843 & - & HQ326943 & - & - & - & [58] \\
\hline Z. erythrocephala & HKAS52844 & - & HQ326944 & - & - & - & [58] \\
\hline Z. olivaceobrunnea & HKAS52275 & - & HO326947 & HO326875 & - & - & {$[58]$} \\
\hline Z. roseola & HKAS75046 & - & KF112414 & KF112269 & KF112579 & KF112791 & [22] \\
\hline S. luridus & IB2004270 & EF644104 & - & - & - & - & [59] \\
\hline S. luridus & 18902 & JF907802 & - & - & - & - & [60] \\
\hline S. luridus & 18182 & JF907793 & - & - & - & - & {$[60]$} \\
\hline S. luridus & Blu3 & AY278765 & _- & - & - & - & {$[61]$} \\
\hline S. luridus & AMB12636 & KC734542 & _- & - & _- & - & [13] \\
\hline S. luridus & AMB12638 & КC734544 & - & - & - & - & [13] \\
\hline S. luridus & TL-6877 & AJ889930 & - & - & - & - & \\
\hline S. luridus & TL-6877 & UDB000077 & _- & - & - & - & \\
\hline S. luridus & 1968 & AY278766 & - & - & - & - & [61] \\
\hline S. luridus & BL2-VII-10 & JQ685714 & - & - & - & - & [61] \\
\hline S. luridus & AT-04 & UDB002401 & - & - & - & - & \\
\hline S. luridus & UP12 & DQ658866 & - & - & - & - & [62] \\
\hline S. luridus & 17696 & JF907789 & - & - & - & - & {$[60]$} \\
\hline S. luridus & BL1-VII-09 & JQ685715 & - & - & - & - & \\
\hline S. luridus & MA-Fungi 47706 & AJ419191 & _- & _- & - & _- & [63] \\
\hline
\end{tabular}


Table 1. Cont.

\begin{tabular}{|c|c|c|c|c|c|c|c|}
\hline Taxon & Voucher ID & ITS & $28 S$ & TEF1 & RPB1 & RPB2 & References \\
\hline S. mendax & AMB12632 & KC734547 & - & - & - & - & [13] \\
\hline S. mendax & AMB12633 & КС734548 & - & - & - & - & [13] \\
\hline S. mendax & AMB12634 & КС734543 & - & - & - & - & [13] \\
\hline S. mendax & AMB12635 & KC734545 & - & - & - & - & [13] \\
\hline S. mendax & AMB12637 & KC734540 & - & - & - & - & [13] \\
\hline S. mendax & AMB12640 & KC734541 & - & - & - & - & [13] \\
\hline Boletus luridus & UF107 & HM347662 & - & - & - & - & \\
\hline B. amygdalinus & src 491 & DQ974705 & - & - & - & - & [64] \\
\hline B. comptus & 17827 & JF907791 & - & - & - & - & [60] \\
\hline B. comptus & AMB12639 & KC734539 & - & - & - & - & [13] \\
\hline B. queletii & 17196 & JF907784 & - & - & - & - & [60] \\
\hline B. queletii & 17208 & JF907785 & - & - & - & - & [60] \\
\hline B. queletii & AMB12641 & KC734546 & - & - & - & - & [13] \\
\hline B. queletii & JV01-231 & UDB000760 & - & - & - & - & \\
\hline N. erythropus & MA-Fungi 47702 & AJ419188 & - & - & - & - & [63] \\
\hline N. erythropus & $\begin{array}{l}\text { BOER TO_2 } \\
(\text { AAM630/06) }\end{array}$ & FM958177 & - & - & - & - & \\
\hline N. erythropus & UF278 & HМ347644 & - & - & - & - & \\
\hline N. erythropus & UF276 & HM347643 & - & - & - & - & \\
\hline N. erythropus & UF269 & HM347665 & - & - & - & - & \\
\hline N. erythropus & DG05-54 & UDB001523 & - & - & - & - & \\
\hline N. erythropus & SU46 & DQ131633 & - & - & - & - & [65] \\
\hline N. erythropus & SU47 & DQ131634 & - & - & - & - & {$[65]$} \\
\hline N. erythropus & Daniels 582 & AJ496595 & - & - & - & - & [63] \\
\hline Caloboletus calopus & AT1998059 & UDB000659 & - & - & - & - & \\
\hline Ca. radicans & TUF106003 & UDB003224 & - & - & - & - & \\
\hline Bu. fuscoroseus & AH96025 & UDB000649 & - & - & - & - & \\
\hline Bu. fuscoroseus & AT1996017 & UDB000652 & - & - & - & - & \\
\hline Bu. fechtneri & AT2003097 & UDB000703 & - & - & - & - & [21] \\
\hline $\begin{array}{l}\text { Imperator } \\
\text { rhodopurpureus }\end{array}$ & AT1996058 & UDB000654 & - & - & - & - & \\
\hline R. pulchrotinctus & GS0860 & UDB000407 & - & - & - & - & \\
\hline R. satanas & AT1998051 & UDB000415 & - & - & - & - & \\
\hline R. rubrosanguineus & GS0405 & UDB000410 & - & - & - & - & \\
\hline R. rhodoxanthus & AT2000182 & UDB001116 & - & - & - & - & \\
\hline $\begin{array}{l}\text { Cyanoboletus } \\
\text { pulverulentus }\end{array}$ & RT00004 & EU819502 & - & - & - & - & \\
\hline $\begin{array}{l}\text { Cyanoboletus } \\
\text { pulverulentus }\end{array}$ & AH97030 & UDB000408 & - & - & - & - & {$[66]$} \\
\hline B. aestivalis & AT2004040 & UDB001113 & - & - & - & - & \\
\hline B. aereus & AT2000198 & UDB000943 & - & - & - & - & \\
\hline
\end{tabular}

\section{Results}

\subsection{Molecular Phylogeny}

The four-locus dataset $(28 \mathrm{~S}+r p b 1+r p b 2+$ tef 1$)$ of Tengioboletus (Supplementary File S1) contained 33 sequences and $3000 \mathrm{bp}$ nucleotide sites. The alignment was submitted to TreeBASE (http:/ / purl.org/phylo/treebase/phylows/study/TB2:S29030, accessed on 15 January 2022). Because the ML tree's topology was the same as the BI tree's topology, only the ML tree was shown (Figure 1). Xanthoconium affine (Peck) Singer and Xanthoconium porophyllum $\mathrm{G}$. Wu \& Zhu L. Yang were chosen as outgroups. The phylogenetic tree showed that two T. subglutinosus sequences formed an independent lineage, with bootstrap proportions $(\mathrm{BP})=100$ and posterior probability $(\mathrm{PP})=1$, and formed a sister group with T. glutinosus $(\mathrm{BP}=100, \mathrm{PP}=1)$.

The four-locus dataset (ITS $+28 \mathrm{~S}+t e f 1+r p b 2)$ of Butyriboletus (Supplementary File S2) consisted of 58 taxa and 3011 nucleotide sites (Figure 2). The alignment was submitted to TreeBASE (http:/ / purl.org/phylo/treebase/phylows/study/TB2:S29034, accessed on 15 January 2022). Baorangia pseudocalopus (Hongo) G. Wu \& Zhu L. Yang was chosen as the outgroup. The phylogram indicated our collections-HMJAU59471, HMJAU59470, and HMJAU60200, HMJAU60201 — were grouped together respectively and formed two independent lineages with high support value $(\mathrm{BP}=100, \mathrm{PP}=1$ and $\mathrm{BP}=99, \mathrm{PP}=1)$.

The four-locus dataset $(28 \mathrm{~S}+r p b 1+r p b 2+t e f 1)$ of Suillellus (Supplementary File S3) involved 64 taxa and 3048 bp sites. Tylopilus alpinus Y.C. Li \& Zhu L. Yang and Tylopilus atripurpureus (Corner) E. Horak were selected as outgroups. The alignment was submitted to TreeBASE (http:/ / purl.org/phylo/treebase/phylows/study/TB2:S29037, accessed on 15 January 2022). The phylogram showed our species belongs to Suillellus (Figure 3). It 
formed an independent sister clade to Suillellus subamygdalinus Kuan Zhao \& Zhu L. Yang, with a solid support $(\mathrm{BP}=92, \mathrm{PP}=1)$. The ITS dataset of Suillellus (Supplementary File S4) consisted of 55 taxa and 885 bp sites. Boletus aestivalis (Paulet) Fr. and Boletus aereus Bull. were chosen as outgroups (Figure 4). The alignment was submitted to TreeBASE (http:/ / purl.org/phylo/treebase/phylows/study/TB2:S29087, accessed on 15 January 2022). The phylogram showed that our species was close to Suillellus comptus (Simonini) Vizzini, Simonini \& Gelardi and formed an independent and robust support clade $(\mathrm{BP}=98$, $\mathrm{PP}=1$ ).

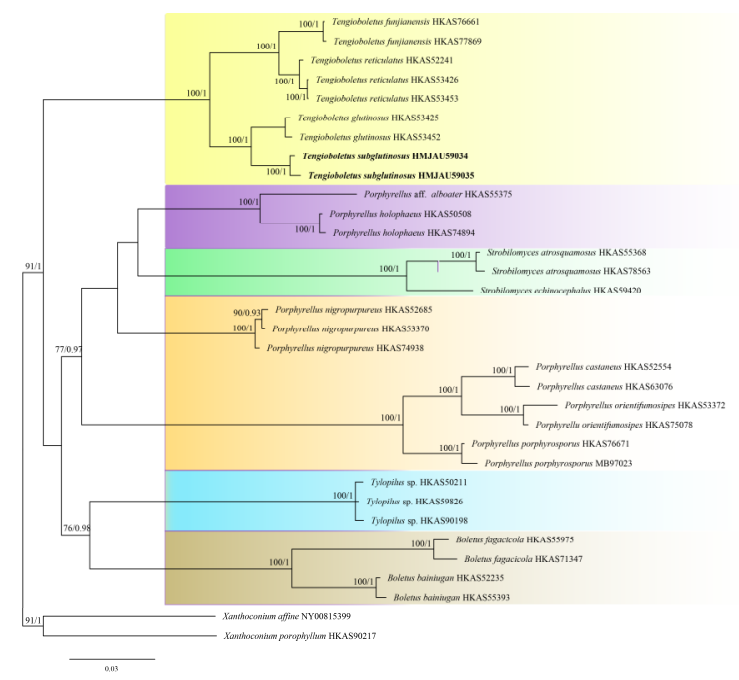

\author{
Tengioboletus \\ Porphyrellus? \\ Strobilomyces \\ Porphyrellus \\ Tylopilus \\ Boletus
}

Figure 1. Phylogenetic analysis of Tengioboletus inferred from ML analysis. BP value (>70) and PP value $(>8)$ are shown around branches. Our new species sequences are indicated in bold.

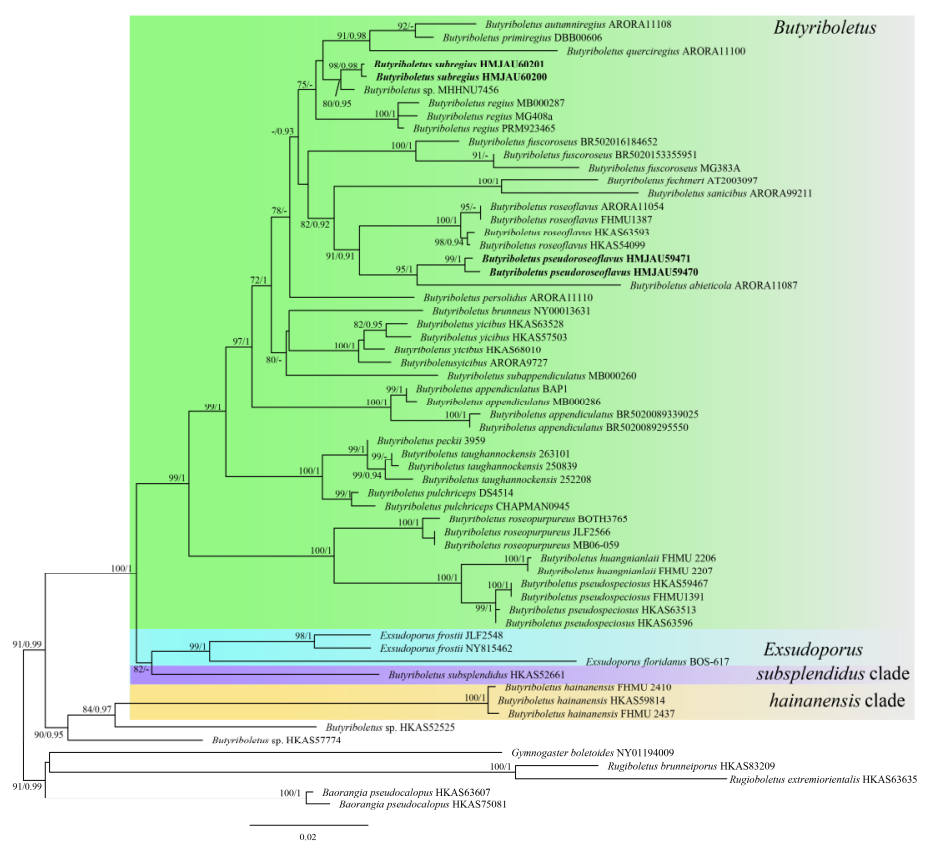

Figure 2. Phylogenetic analysis of Butyriboletus inferred from ML analysis. BP value (>70) and PP value (>9) are shown around branches. Our new species sequences are indicated in bold. 


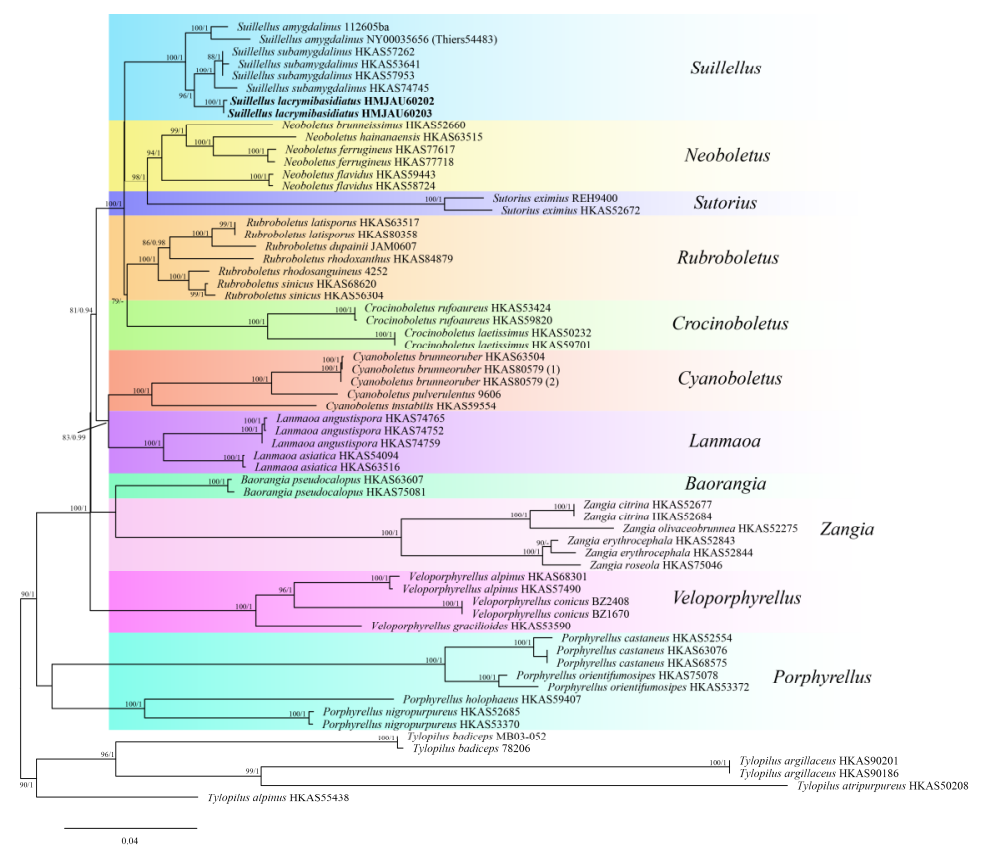

Figure 3. Phylogenetic analysis of Suillellus inferred from ML analysis based on the multi-locus dataset. BP value (>70) and PP value (>9) are shown around branches. Our new species sequences are indicated in bold.

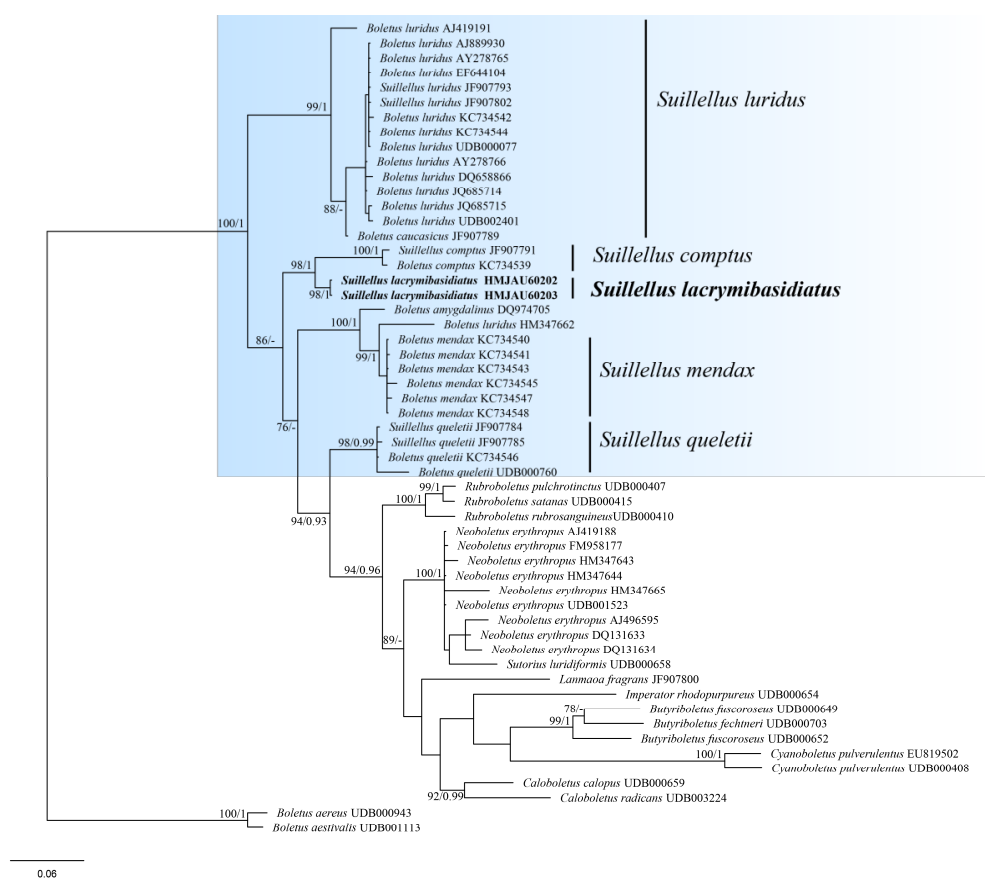

Figure 4. Phylogenetic analysis of Suillellus inferred from Bayes inference analysis based on ITS dataset. BP value (>70) and PP value (>9) are shown around branches. Our new species sequences are indicated in bold.

\subsection{Taxonomy}

Butyriboletus pseudoroseoflavus Yang Wang, Bo Zhang \& Yu Li, sp. nov. Mycobank No.: 842167.

Figures 5e, 6 and 7d. 


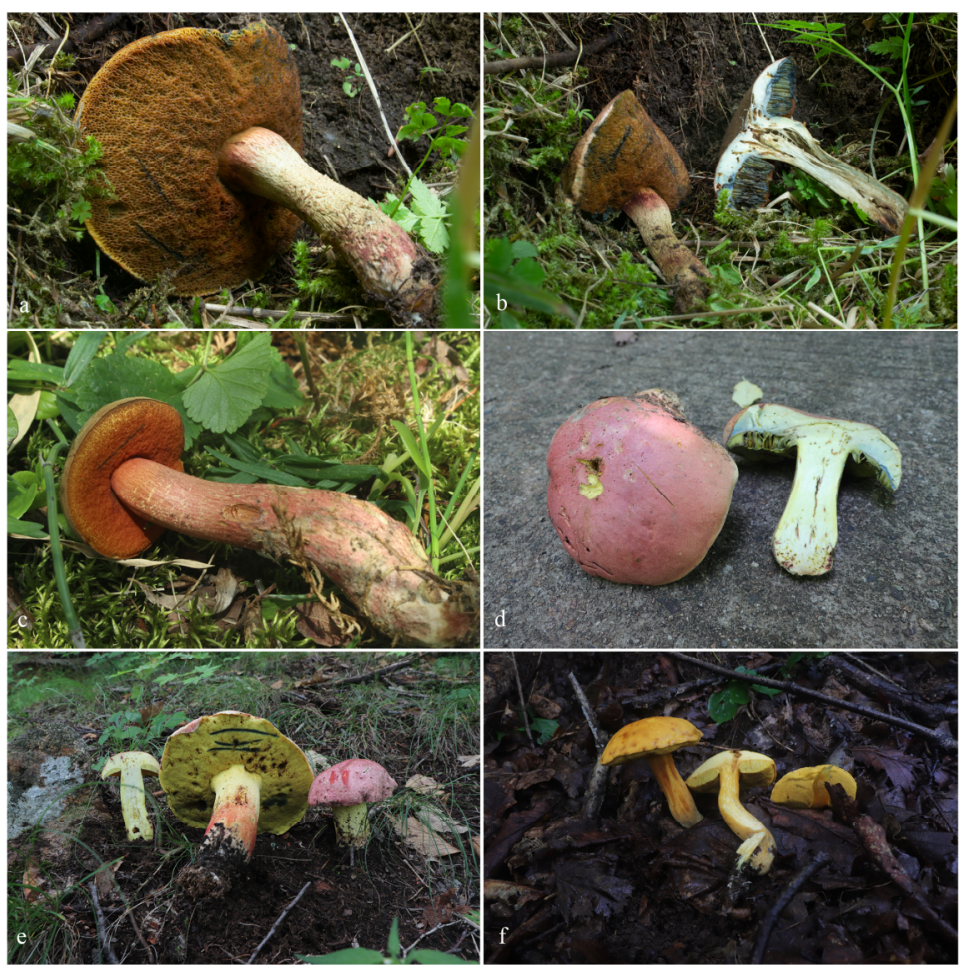

Figure 5. Basidiomata of boletes. (a-c) Suillellus lacrymibasidiatus; (d) Butyriboletus subregius; (e) Butyriboletus pseudoroseoflavus (e from HMJAU 59470); (f) Tengioboletus subglutinosus (f from HMJAU 59037). (a-c) Photos by Yang Wang; (d-f) Photos by Yong-Lan Tuo.

Etymology. The epithet "pseudoroseoflavus" refers to its similarity to B. roseoflavus.

Holotypus. China. Jilin Province, Jian city, Wunvfeng National Forest Park, $125^{\circ} 34^{\prime} 33^{\prime \prime}$ E, $40^{\circ} 52^{\prime} 7^{\prime \prime}$ N, under Quercus mongolica, on dark-brown soil, alt. $1210 \mathrm{~m}, 16$ August 2019, Gu Rao 383 (HMJAU 59471!).

Basidiomata large. Pileus $13.5-17.0 \mathrm{~cm}$ in diameter, hemispherical to applanate, with slightly or distinctly appendiculate margin, sometimes incurved at the margin when young; surface tomentose, pink (12A4) to greyish rose (11B5), context 1.1-1.8 cm thick, light yellow (1A5), unchanging in color when injured. Hymenophore adnate to decurrent, surface greenish yellow (1B8), becoming greenish blue (23B8) quickly when bruised; pores round to angular, ca. $1-3 / \mathrm{mm}$; tubes $1.5-1.7 \mathrm{~cm}$ long, concolorous with pore surface, unchanging color when injured. Stipe 9.0-14.7 $\times 2.0-3.6 \mathrm{~cm}$, subcylindrical, robust, yellow on the upper portion, vivid red (10A8) downwards, surface almost wholly covered yellow (2B8) reticulation or at least upper two thirds; basal mycelium white.

Basidiospores (60/3/2) (7.0) 10.2-10.6-11.0 (16.0) × (2.0) 3.1-3.2-3.7 (4.0) $\mu \mathrm{m}, \mathrm{Q}=(2.0)$ 2.5-4.6 (5.3), $\mathrm{Qm}=3.30 \pm 0.58$, elongate oblong to subfusoid, inequilateral with a suprahilar depression in side view, light yellow in $5 \% \mathrm{KOH}$, smooth. Hymenophoral trama boletoid, hyphae cylindrical, 2.5-10 $\mu \mathrm{m}$ wide. Basidia clavate, thin-walled, 16.0-33.0 $\times 2.0-10.0 \mu \mathrm{m}$, 2- and 4-spored. Cheilocystidia 31.5-50.0 $\times 5.0-10.0 \mu \mathrm{m}$, narrowly lageniform, thin-walled, pale yellow in 5\% KOH. Pleurocystidia 37.5-62.5 $\times 5.0-11.5 \mu \mathrm{m}$, similar to cheilocystidia in shape. Pileipellis trichodermium, filamentous hyphae 1.7-7.5 $\mu \mathrm{m}$ wide. Stipitipellis fertile, hymeniform with thin-walled and inflated terminal cells $(13.8-26.0 \times 6.8-13.8 \mu \mathrm{m})$. Stipe trama composed of parallel hyphae 2.5-7.5 $\mu \mathrm{m}$ wide. Clamp connections not observed.

Habitat: solitary or scattered on a dark-brown soil of Quercus mongolica forest.

Known distribution: currently, only known from Jilin province, China.

Additional collection examined: China. Jilin Province, Jian city, Wunvfeng National Forest Park, $125^{\circ} 34^{\prime} 33^{\prime \prime}$ E, $40^{\circ} 52^{\prime} 7^{\prime \prime} \mathrm{N}$, under Quercus mongolica, on dark-brown soil, alt. 950 m, 5 August 2020, Yong-Lan Tuo 274 (HMJAU 59470). 

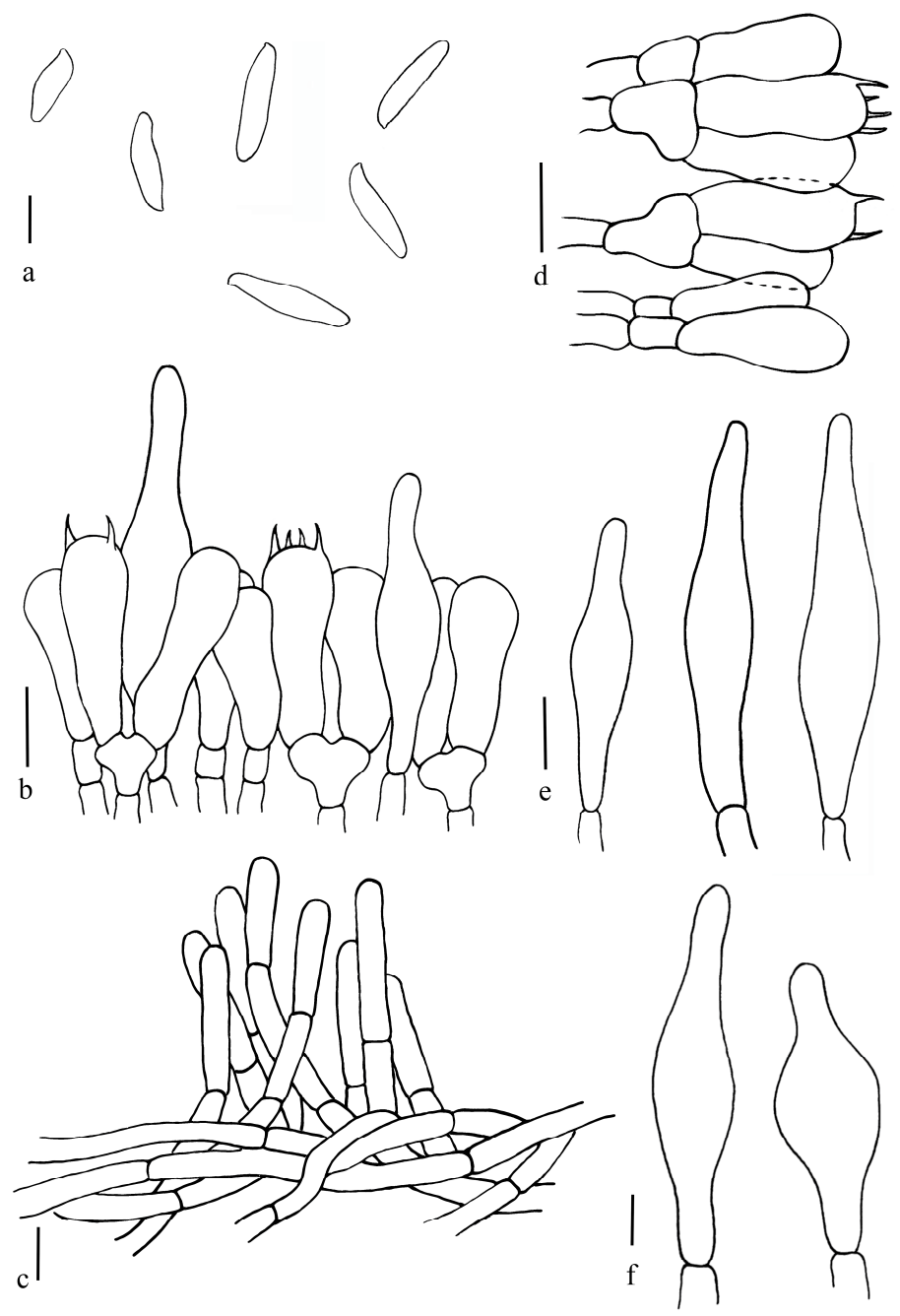

Figure 6. Butyriboletus pseudoroseoflavus. (a) Basidiospores; (b) Basidia and pleurocystidia; (c) Pileipellis; (d) Stipitipellis; (e) Pleurocystidia; (f) Cheilocystidia. Scale bars: $(\mathbf{b}-\mathbf{e})=10 \mu \mathrm{m} ;(\mathbf{a}, \mathbf{f})=5 \mu \mathrm{m}$.

Notes: Butyriboletus pseudoroseoflavus is characterized by a pink to greyish rose pileus, greenish yellow pores changing to greenish blue when bruised, a stipe surface almost wholly covered with yellow reticulation, a stipe of unchanging color when injured, and large and narrow basidiospores. Morphologically and phylogenetically, Bu. pseudoroseoflavus is similar to Bu. roseoflavus (Hai B. Li \& Hai L. Wei) D. Arora \& J.L. Frank, which was initially described in specimens from eastern China (Zhejiang province) and southwestern China (Yunnan province) by Li et al. [67]. However, Bu. pseudoroseoflavus differs from $\mathrm{Bu}$. roseoflavus in its adnate to decurrent hymenophore and its relatively larger and narrower basidiospores, with a more considerable $Q$ value and pleurocystidia larger than cheilocystidia [5]. In morphological features, Bu. pseudoroseoflavus is also similar to Bu. cepaeodoratus (Taneyama \& Har. Takah.) Vizzini \& Gelardi, Bu. roseogriseus (Šutara, M. Graca, M. Kolařík, Janda \& Kř́ž) Vizzini \& Gelardi, Bu. primiregius D. Arora \& J.L. Frank, Bu. regius (Krombh.) D. Arora \& J.L. Frank., and Bu. fuscoroseus (Smotl.) Vizzini \& Gelardi, but the pileus of $B u$. cepaeodoratus always has a duller color, its stipe stains blue when injured, and its basidiospores are broader than those of Bu. pseudoroseoflavus [68]. Both stipe and context of $\mathrm{Bu}$. roseogriseus and $\mathrm{Bu}$. primiregius turn blue when injured, and have broader basidiospores, $\mathrm{Q}=(1.95) 2.20-2.42(2.57)$ and $\mathrm{Q}=3.5$, respectively $[32,56]$. The pores of $B u$. regius are unchanging to blue when bruised; the stipe is usually ventricose when young, showing at the base rare faintly reddish or purplish spots, with basidiospores weakly dextrinoid [69]. Butyriboletus fuscoroseus is characterized by its brown-pink, reddish brown, or purplish 
brown pileus, decurrent hymenophore, stipe staining blue when bruised or cut, and the narrow basidiospores [56]. Phylogenetically, Bu. pseudoroseoflavus is similar to Bu. abieticola. However, Bu. abieticola is characterized by a light rose-colored pileus, with tan-colored spots interspersed, a white context, a hymenium dextrinoid, and hyaline spiral incrustations on most hyphae [70]. Reference Table 2 provides the critical characteristics distinguishing $\mathrm{Bu}$. pseudoroseoflavus from other species in China.

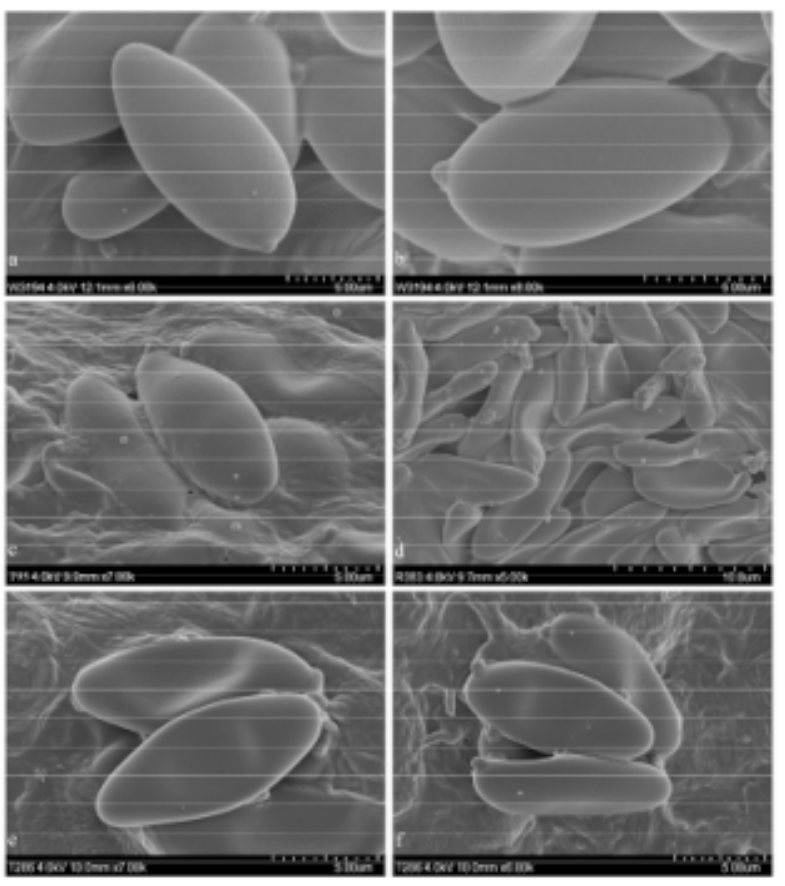

Figure 7. Basidiospores observed in the SEM. (a,b) Suillellus lacrymibasidiatus; (c) Butyriboletus subregius; (d) Butyriboletus pseudoroseoflavus; (e,f) Tengioboletus subglutinosus.

Butyriboletus subregius Yang Wang, Bo Zhang \& Yu Li, sp. nov.

Mycobank No.: 842517.

Figures $5 \mathrm{~d}, 7 \mathrm{c}$ and 8.

Etymology::"sub" means "near," named because it is similar to B. regius.

Holotypus: China. Jilin Province, Jian city, Wunvfeng National Forest Park, $125^{\circ} 34^{\prime} 33^{\prime \prime}$ E, $40^{\circ} 52^{\prime} 7^{\prime \prime} \mathrm{N}$, under Quercus mongolica, on dark-brown soil, alt. $1050 \mathrm{~m}$, 7 July 2020, Yong-Lan Tuo 95 (HMJAU 60200!).

Basidiomata middle to large. Pileus $7.0-13.0 \mathrm{~cm}$ in diameter, hemispherical or broadly hemispherical at maturity, with distinctly appendiculate margin initially, surface dry, covered with weak or distinct tomentum, pastel pink (11A4-5), context yellowish green (30A6), turning blue when cut. Hymenophore weakly decurrent, covered with a layer of whitish mycelium (1A1) when young, surface yellowish green (29A6), bluing when bruised, pores angular to nearly round, ca. $4-5 / \mathrm{mm}$; tubes concolorous with hymenophore surface, about $1.1 \mathrm{~cm}$ long, turning blue when cut. Stipe 11.0-14.5 $\times 4.4-5.0 \mathrm{~cm}$, subcylindrical or enlarged downwards, yellowish green (29A6) at maturity, covered with pastel red stains when young, upper $2 / 3$ portion covered with yellowish green (29A6) reticulation, staining blue when bruised, context yellowish green (30A6), changing weakly to blue when cut. 
Table 2. Morphological comparisons of Butyriboletus pseudoroseoflavus sp. nov. and Butyriboletus subregius sp. nov. with other Butyriboletus spp. reported in China.

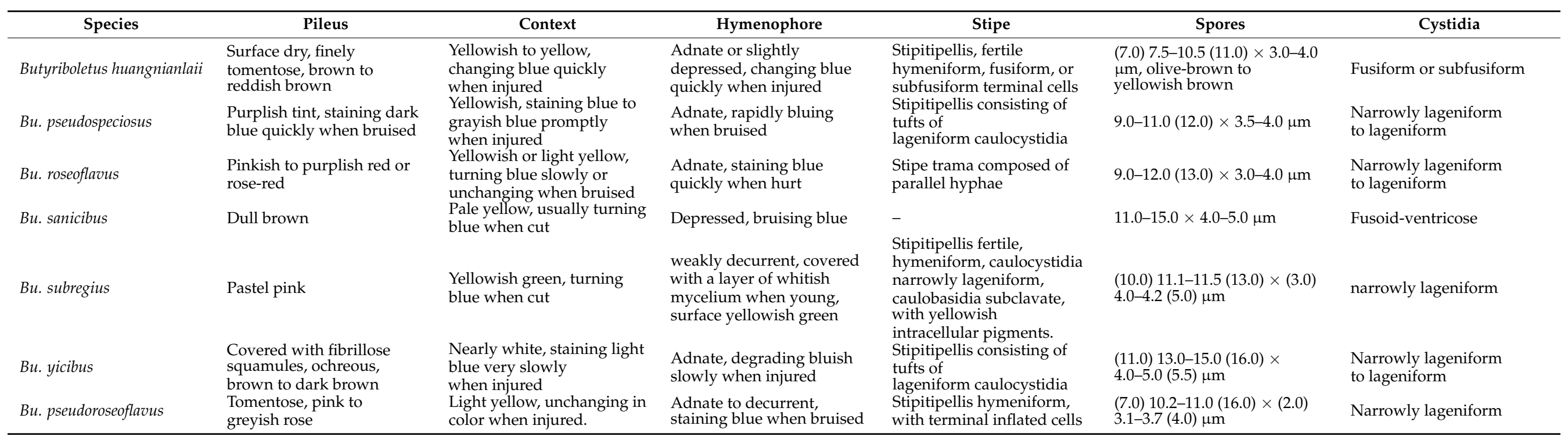




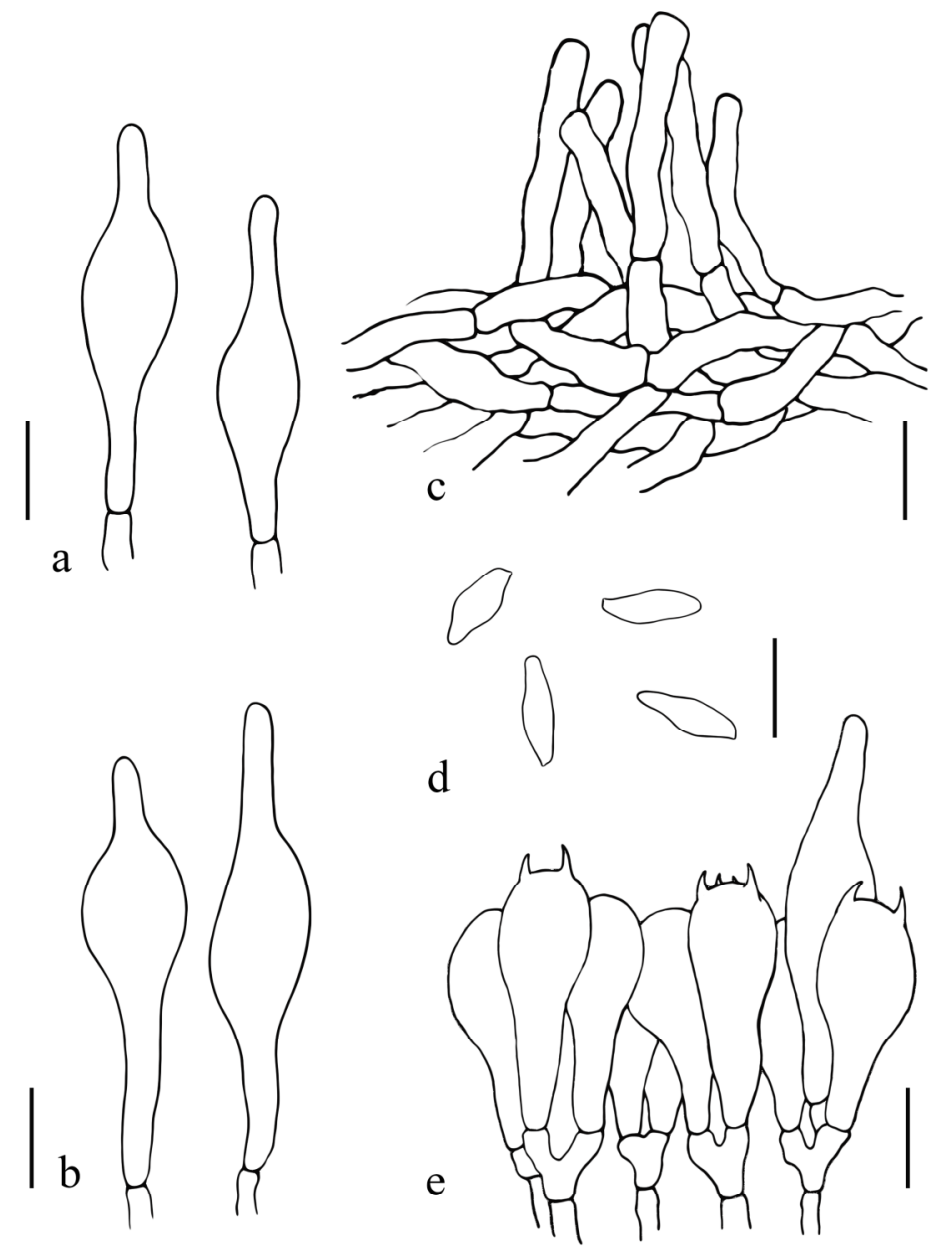

Figure 8. Butyriboletus subregius. (a) Pleurocystidia; (b) Cheilocystidia; (c) Pileipellis; (d) Basidiospores. (e) Pleurocystidia and basidia. Scale bars: $10 \mu \mathrm{m}$.

Basidiospores (60/2/2) (10.0) 11.1-11.3-11.5 (13.0) × (3.0) 4.0-4.1-4.2 (5.0) $\mu \mathrm{m}$, $\mathrm{Q}=(2.22)$ 2.40-3.00 (4.00), $\mathrm{Qm}=2.76 \pm 0.31$, subfusoid to subcylindrical, inequilateral with a suprahilar depression in side view, brownish yellow in $5 \% \mathrm{KOH}$, smooth. Basidia 21.0-36.0 $\times 8.0-12.5 \mu \mathrm{m}$, clavate, 2- and 4-spored. Hymenophoral trama boletoid, composed of hyphae 4-7 $\mu \mathrm{m}$ in diameter. Pleurocystidia 36.3-56.7 $\times 7.0-14.6 \mu \mathrm{m}$, narrowly lageniform, thin-walled, yellowish in 5\% KOH. Cheilocystidia 22.0-50.5 $\times 5.5-12.4 \mu \mathrm{m}$, narrowly lageniform. Pileipellis a trichodermium, composed of filamentous hyphae, 3.0-6.5 $\mu \mathrm{m}$ wide. Stipitipellis fertile, hymeniform, caulocystidia $23.0-43.5 \times 9.0-12.5 \mu \mathrm{m}$, narrowly lageniform, caulobasidia 17.2-32.0 ×6.2-8.0 $\mu \mathrm{m}$, subclavate, with yellowish intracellular pigments. Clamp connections not observed.

Habitat: solitary or scattered on a dark-brown soil of Quercus mongolica forest.

Known distribution: currently, only known from Jilin province, China.

Additional collection examined: China. Jilin Province, Jian city, Wunvfeng National Forest Park, under Quercus mongolica, on dark-brown soil, alt. 1050 m, 10 August 2019, Yong-Lan Tuo 198 (HMJAU 60201).

Notes: Butyriboletus subregius is characterized by a pastel pink pileus, a yellowish green stipe covered with reticulation of the same color and staining blue when the hymenophore and stipe are bruised. Morphologically and phylogenetically, Bu. subregius resembles $B u$. autumniregius, Bu. primiregius, Bu. querciregius, Bu. regius and Bu. fuscoroseus. However, $B u$. autumniregius is distinguished by its autumn fruiting season, a stipe that commonly has dark red stains toward the base, and longer spores with a larger $Q$ value [33]; Bu. primiregius is characterized by its late spring season, and a pileus tending to dingy olive-brown as it 
ages or exposed in sunlight [33]; Bu. querciregius differs from $B u$. subregius in its mycorrhizal host, the dull color of a pileus, relatively longer spores with larger $Q$ value [33]; Bu. regius is different from $B u$. subregius in its pileus covered with distinct scales with aging, a context usually not bluing when cut, and spores longer with larger $Q$ value [69]. Butyriboletus fuscoroseus is different from Bu. subregius in its brown-pink, reddish brown, or purplishbrown pileus, fine reticulation covered only on the upper half of stipe and context of stipe strongly bluing when cut [56]. Reference Table 2 provides the critical characteristics distinguishing $\mathrm{Bu}$. subregius from other species in China.

Tengioboletus subglutinosus Yang Wang, Bo Zhang \& Yu Li, sp. nov.

Mycobank No.: 842168.

Figures $5 \mathrm{f}, 7 \mathrm{e}, \mathrm{f}$ and 9.
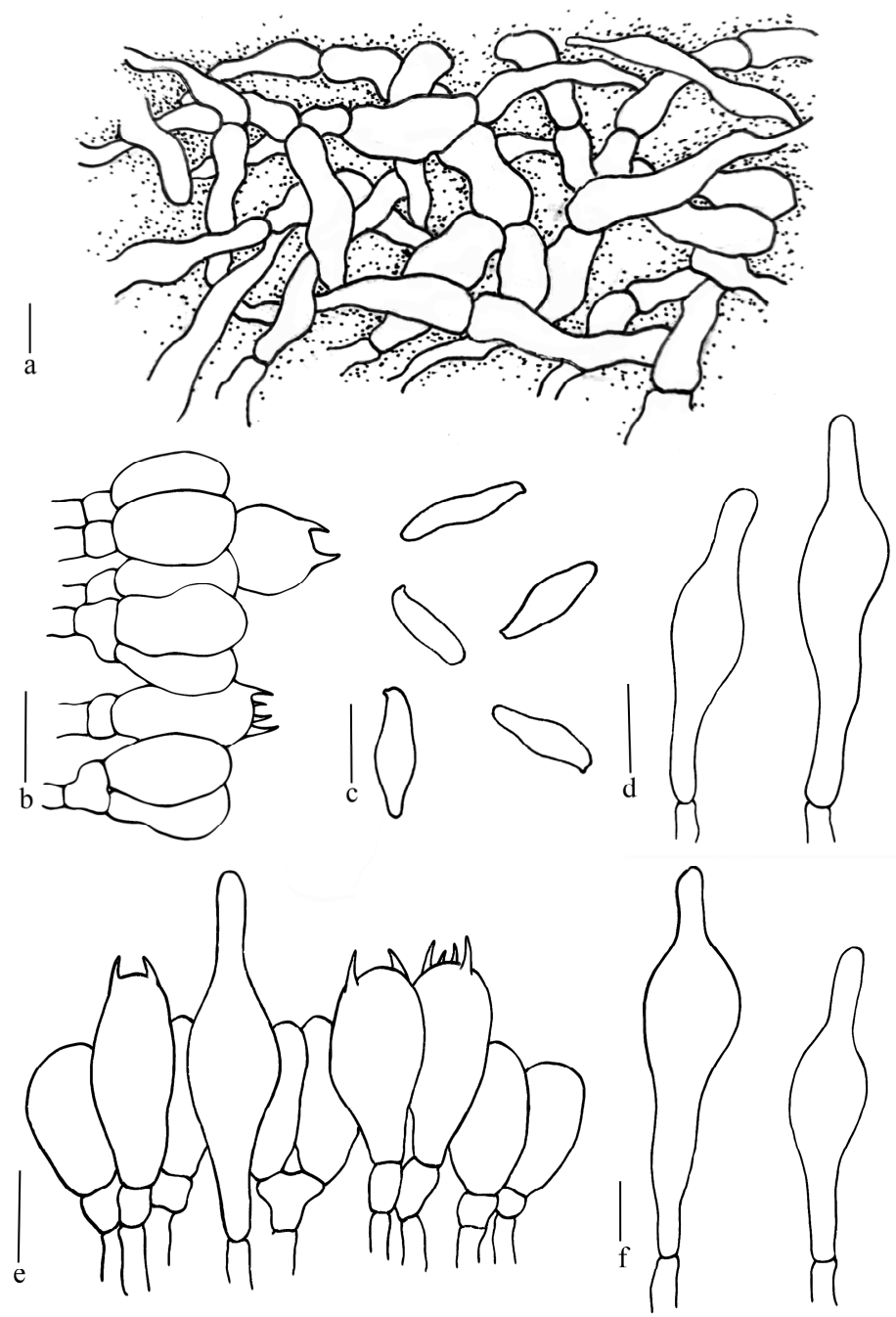

Figure 9. Tengioboletus subglutinosus. (a) Pileipellis; (b) Stipitipellis; (c) Basidiospores; (d) Cheilocystidia; (e,f) Pleurocystidia and basidia. Scale bars: $10 \mu \mathrm{m}$.

Etymology: "sub" means "near," named because it is similar to T. glutinosus.

Holotypus: China. Jilin Province, Jian city, Wunvfeng National Forest Park, $125^{\circ} 34^{\prime} 33^{\prime \prime}$ E, $40^{\circ} 52^{\prime} 7^{\prime \prime} \mathrm{N}$, under Quercus mongolica, on dark-brown soil, alt. $650 \mathrm{~m}, 6$ August 2020, Y. L. Tuo 293 (HMJAU 59035!).

Basidiomata medium to large. Pileus $6.5-9.0 \mathrm{~cm}$ in diameter, hemispherical to applanate, surface brownish yellow (5C8) to yellowish brown (5D8), glabrous, viscid when wet, context deep yellow (4A8), $0.6-1.5 \mathrm{~cm}$ thick, color unchanging when cut; hymenophore sinuate to decurrent; tubes up to $1.3 \mathrm{~cm}$ long, vivid yellow (3A8), changing to indistinct 
blue erratically or unchanging color when cut; hymenophore surface concolorous with tubes or olive yellow (3C8), staining blue when bruised; pores angular, ca. $2-3 / \mathrm{mm}$. Stipe $7.2-16.0 \times 1.4-2.2 \mathrm{~cm}$, central, clavate to subcylindrical, solid, sometimes tapered downwards, surface concolorous with pileus surface, covered with fine reticulation at apex, context deep yellow (4A8), color unchanging when cut; basal mycelium yellow (3B8).

Basidiospores (60/2/1) (10.0) 11.5-11.7-11.9 (13.0) × (4.0) 4.2-4.3-4.4 (6.0) $\mu \mathrm{m}[\mathrm{Q}=(1.70)$ 2.00-3.17 (3.25) $2.75 \pm 0.3$ ], elongate ellipsoid and inequilateral in side view, with distinctly suprahilar depression; greenish yellow (1A8) in 5\% $\mathrm{KOH}$, smooth. Hymenophoral trama of the intermediate type between phylloporoid and boletoid types. Basidia 19.0-42.0 $\times$ 6.0-13.0 $\mu \mathrm{m}$, clavate, 2- and 4-spored, hyaline in 5\% KOH. Pleurocystidia scattered, 45.0$65.0 \times 9.0-15.0 \mu \mathrm{m}$, fusoid-ventricose to broadly fusoid-ventricose, with subacute apex or long beak, thin-walled. Cheilocystidia 36.0-50.0 $\times 7.5-10.5 \mu \mathrm{m}$, similar to pleurocystidia in shape. Pileipellis an interwoven ixotrichodermium, with inflated terminal cells $28.5-57.0 \times$ 15.0-23.0 $\mu \mathrm{m}$. Stipitipellis fertile, hymeniform, with subglobose to globose terminal cells, and scattered clavate basidia.

Habitat: solitary or scattered on a dark-brown soil of Quercus mongolica forest.

Known distribution: currently, only known from Jilin province, China.

Additional collections examined: China. Jilin Province, Jian city, Wunvfeng National Forest Park, under Quercus mongolica, on dark-brown soil, alt. 900 m, 6 August 2020, Yong-Lan Tuo 286 (HMJAU 59034); alt. 750 m, 11 August 2020, Yong-Lan Tuo 344 (HMJAU 59036); alt. 650 m, 23 August 2020, Yong-Lan Tuo 471 (HMJAU 59037).

Notes: Tengioboletus subglutinosus is characterized by a hymenophore surface staining blue when bruised, a pileipellis in the form of an ixotrichodermium, with inflated or clavated terminal cells. Morphologically and phylogenetically, T. subglutinosus is similar to T. glutinosus. However, T. subglutinosus is different due to its hymenophoral surface staining blue when bruised, a hymenophore sinuate to decurrent, a stipe with reticulations at the apex, and narrower spores [5]. Tengioboletus fujianensis differs from T. subglutinosus in its hymenophoral surface staining brown when bruised, prominently reticulation nearly to the stipe base and hymenophoral trama boletoid [34]. Basidiomata of T. reticulatus show a distinct olive-brown, brown-to dark-brown pileus, shorter hymenophore of unchanging color when bruised, a distinct reticulation covering stipe, and a pileipellis trichodermium, not ixotrichodermium [5].

Suillellus lacrymibasidiatus Yang Wang, Bo Zhang \& Yu Li, sp. nov.

Mycobank No.: 842518.

Figures 5a-c, 7a,b and 10 .

Etymology: "lacrymibasidiatus" means most of its basidia seem lacrymoid.

Holotypus: China. Xinjiang Uygur Autonomous Region: Ili Kazakh Autonomous Prefecture, Xinyuan county, $84^{\circ} 31^{\prime} 20^{\prime \prime}$ E, $43^{\circ} 15^{\prime} 43^{\prime \prime} \mathrm{N}$, under Pinus schrenkiana, on light grayish brown loess, alt. 1899 m, 3 August 2021, W3194 (HMJAU 60202!).

Basidiomata medium. Pileus $4.1-8.2 \mathrm{~cm}$ in diameter, hemispherical then applanate, surface oak brown (5D6) when young, brownish orange (6C6) at maturity, weakly tomentose, context yellowish white (1A2), $0.4-0.9 \mathrm{~cm}$ thick, turning blue when cut. Hymenophore adnexed, surface tomato red (8C8) when young, brick red (7D7) at maturity, bluing quickly when injured, pores angular, ca. $1-3 / \mathrm{mm}$; tubes up to $1.3 \mathrm{~cm}$ long, sulfur yellow (1A5), bluing promptly when cut. Stipe 7.2-7.4 × 1.7-2.0 cm, subcylindrical, relatively slender at middle part or attenuate downwards, surface red (10A6) when young, finely longitudinally reticulated over the apex, color of surface fading to yellow and covered with distinct squamules at the middle part in ages, context pastel green (30A4), turning blue when cut; basal mycelium white.

Basidiospores (60/2/2) (11.6) 14.5-14.7-15 (17.0) × (6.7) 7.7-7.8-7.9 (9.0) $\mu \mathrm{m}, \mathrm{Q}=1.5-2.1$, $\mathrm{Qm}=1.9 \pm 0.1$, subamygdaloid to broadly ellipsoid, brown in 5\% $\mathrm{KOH}$, smooth, neither amyloid nor dextrinoid. Hymenophoral trama boletoid type, composed of 2.0-16.5 $\mu \mathrm{m}$ wide hyphae, amyloid. Basidia 20.8-38.5 × 13.0-20.1 $\mu \mathrm{m}$, lacrymoid, 2- and 4-spored, hyaline in $5 \% \mathrm{KOH}$. Pleurocystidia and cheilocystidia not observed. Pileipellis a trichoder- 
mium, composed of 5.0-9.5 $\mu \mathrm{m}$ wide, yellowish brown, inamyloid hyphae. Stipitipellis hymeniderm, terminal cells inflated, 25.8-61.2 $\times 12.0-15.5 \mu \mathrm{m}$. Hyphae of the flesh in the stipe base amyloid in Melzer's reagent. Clamp connections not observed.

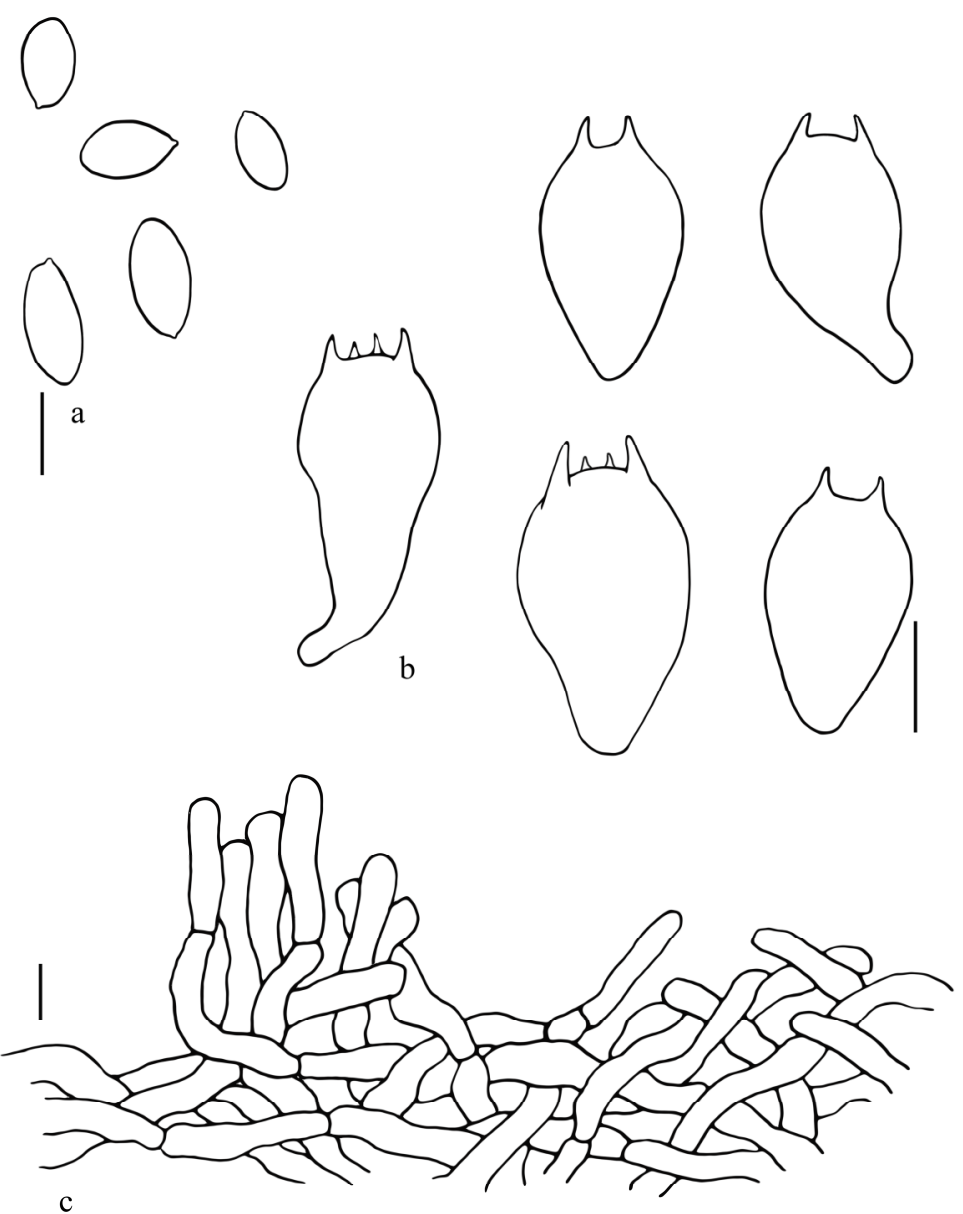

Figure 10. Suillellus lacrymibasidiatus. (a) Basidiospores; (b) Basidia; (c) Pileipellis. Scale bars: $10 \mu \mathrm{m}$.

Habitat: solitary or scattered on a black loam soil of Salix spp. and Populus spp. mixed forest or a light grayish brown loess of Pinus schrenkiana forest.

Known distribution: currently, only known from Xinjiang Uygur Autonomous Region, China.

Additional collection examined: China. Xinjiang Uygur Autonomous Region: Ili Kazakh Autonomous Prefecture, Zhaosu County, $80^{\circ} 42^{\prime} 30^{\prime \prime}$ E, $42^{\circ} 59^{\prime} 37^{\prime \prime} \mathrm{N}$, under river valley with presence of Salix spp. and Populus spp., on black loam soil, alt. $1697 \mathrm{~m}$, 6 August 2021, W3229 (HMJAU 60203).

Notes: Suillellus lacrymibasidiatus is characterized by its oak brown to brownish orange pileus, the context staining blue when injured, and inamyloid basidiospores. Morphologically, S. lacrymibasidiatus is related to S. luridus (Schaeff.) Murrill, S. mendax (Simonini \& Vizzini) Vizzini, Simonini \& Gelardi, S. queletii (Schulzer) Vizzini, Simonini \& Gelardi, and S. subamygdalinus Kuan Zhao \& Zhu L. Yang. S. luridus is characterized by its prominent reticulation on the stipe and smaller basidiospores [71]; S. mendax is different from S. lacrymibasidiatus in its promptly bluing when pileus bruised, value of $\mathrm{Q}$ larger, and basidia clavate [13]; S. queletii can be distinguished from S. lacrymibasidiatus by its stipe wholly covered with fine granulation without reticulation, basidia clavate [72]; S. subamygdalinus is characterized by its basidia clavate [5]. Phylogenetically, S. lacrymibasidiatus is related to S. comptus. However, S. comptus differs from S. lacrymibasidiatus in its stipe surface staining 
blue when bruised, and smaller spores [71]. Among the other morphologically allied boletes, S. adalgisae (Marsico \& Musumeci) N. Schwab [73], S. austrinus (Singer) Murrill [74], S. gabretae (Pilát) Blanco-Dios [75], S. luridiceps Murrill [76], and S. subvelutipes (Peck) Murrill. [77], none of them could represent a possible concurrent of S. lacrymibasidiatus.

A key to worldwide species of Tengioboletus:

1. Pores changing color when bruised

1. Pores unchanging color when bruised

2. Pores staining blue when bruised, pileipellis an ixotrichodermium

2. Pores staining brown when bruised, pileipellis an trichodermium

3. Stipe covered with distinct reticulations, basidiospores larger,

12.0-14.5 $\times 4.5-6.0 \mu \mathrm{m}$, pileipellis an trichodermium

3. Stipe nearly glabrous, basidiospores $10.0-12.0 \times 3.5-4.5 \mu \mathrm{m}$,

pileipellis, an ixotrichodermium

A key to worldwide species of Suillellus s.str.:

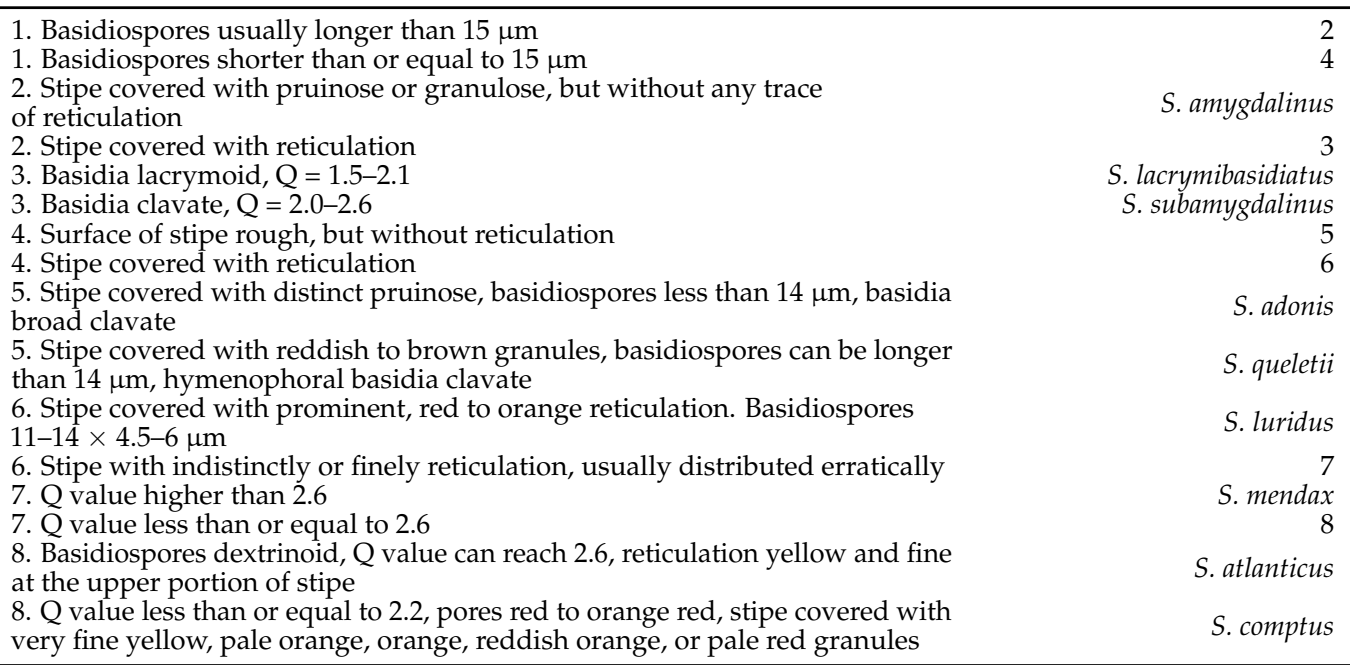

\section{Discussion}

In this study, four new species, Butyriboletus pseudoroseoflavus, Butyriboletus subregius, Tengioboletus subglutinosus, and Suillellus lacrymibasidiatus, were discovered in northern China based on morphological studies and phylogenetic analyses.

Seven species of Butyriboletus were previously reported in China, and all of them were collected from tropical and subtropical regions of China. The two new species of Butyriboletus we proposed here are the first reports of this genus in northern China. Moreover, according to Arora et al. [33], the species diversity of the genus should be more abundant in temperate climes than tropical, subtropical, or boreal ones. Based on this, we presume that northern China may be a species diversity hotspot of Butyriboletus waiting to be explored further.

Butyriboletus subregius is easily confused with $\mathrm{Bu}$. autumniregius, Bu. primiregius, $\mathrm{Bu}$. querciregius, and $\mathrm{Bu}$. regius, morphologically. The primary distinguishing characteristics are the fruiting time and different ecological niches. According to Queiroz [78], these differences mean that the features formerly treated as secondary species criteria are relevant to species delimitation, to the extent that they provide evidence of a lineage separation. Although one ITS sequence of Bu. loyo (Phillippi) Mikšík, was uploaded to the GeneBank [79], the authors did not give a detailed morphological description to prove identification accuracy, so it was excluded from the current phylogeny. However, Bu. loyo is unique within this genus, given its combined morphological characteristics of being equilateral in profile and having red-brown basidiospores and a viscid pileus.

Due to the different color of the hymenophore surface and tubes and the usually vivid red color of basidiomata, Farid et al. [19], Bozok et al. [80], and Biketova et al. [37] all recommended Exsudoporus as a genus separate from Butyriboletus, including B. floridanus (Singer) G. Wu, Kuan Zhao \& Zhu L. Yang, B. frostii (J.L. Russell) G. Wu, Kuan Zhao, \& Zhu L. Yang, and E. permagnificus (Pöder) Vizzini, Simonini, \& Gelard. However, only the result 
of Farid et al. [19] showed that the B. subsplendidus (W.F. Chiu) Kuan Zhao, G. Wu, \& Zhu L. Yang clade has affinity with other Butyriboletus. Our phylogram accords with the findings of Chai [28] and Biketova et al. [37] that B. subsplendidus is a sister to the Exsudoporus clade. We agree with Biketova et al. [37] that Exsudoporus should be elevated to genus status, and B. subsplendidus and B. hainanensis N.K. Zeng, Zhi Q. Liang, \& S. Jiang should separate from Butyriboletus and represent two distinct genera, as their apparently different characteristics from other species of Butyriboletus.

Tengioboletus is a small genus, with only three species previously reported in southern China. Tengioboletus reticulatus was the first species of the genus collected at Liaoning province in northeastern China [81]. In our study, one new species, T. subglutinosus, was also collected at Jilin province in northeastern China. This means a geographical extension of Tengioboletus into temperate zones, which may also indicate a potentially wide distribution, given that their previously known main distribution was subtropical and tropical China. Our study showed that sequences of Tengioboletus formed an independent clade, which corresponded to the findings of $\mathrm{Wu}$ et al. [5] and supported Tengioboletus as a separate genus. As found by Wu et al. [5], Porphyrellus E.-J. Gilbert is a polyphyletic genus in the phylogram (Figure 1); it formed two clades; one clade named "Porphyrellus?" is a sister to Strobilomyces Berk., as was implied by Han et al. [82]. Clarification of the relationships among the genera will require additional specimens and future studies.

Recently, many genera were merged or erected in boletes as part of the development of molecular technology. Wu et al. [5] treated Neoboletus Gelardi, Simonini, \& Vizzini as synonymized with Sutorius Halling, Nuhn, \& N.A. Fechner, based on molecular data. However, Chai et al. [28] studied the morphological characteristics and reconstructed phylogenetic trees of Neoboletus, Sutorius, Costatisporus T.W. Henkel \& M.E. Sm., and Caloboletus Vizzini. They considered that Neoboletus do not belong to Sutorius. Our phylogenetic analyses (Figure 3) confirmed their results.

Rubroboletus Kuan Zhao \& Zhu L. Yang, Neoboletus, Sutorius, and Lanmaoa G. Wu \& Zhu L. Yang shares some characteristics with Suillellus, such as the orange-red surface of the hymenophore and the bluing color change. Nevertheless, none of them has the amyloid hyphae of the context $[5,25,83-85]$. In contrast, Rubroboletus species have a vivid or dark red pileus with rose-to-red reticulation, and the stipes of species of Neoboletus usually have fine dots but never reticulation. The basidiomata of Sutorius always have a dull color and a reddish color change $[28,86]$; the hymenophore of Lanmaoa is thin, with a thickness about $1 / 3-1 / 5$ times that of the pileal context at the position halfway to the pileus center.

Supplementary Materials: The following are available online at https:/ /www.mdpi.com/article/ 10.3390/jof8030218/s1, File S1: Tengioboletus matrix, File S2: Butyriboletus matrix, File S3: Suillellus multigene matrix, File S4: Suillellus ITS matrix.

Author Contributions: Conceptualization, B.Z. and Y.L.; Methodology, Y.W.; Software, X.-M.W. and J.W.; Investigation, Y.W., Y.-L.T., and G.R.; Resources, Y.W., Y.-L.T., G.R., Z.-H.Z., D.-M.W. and N.G.; Data Curation, B.Z. and Y.L.; Writing-Original Draft Preparation, Y.W.; Writing-Review \& Editing, B.Z. and D.D.; Visualization, Y.W.; Supervision, B.Z.; Project Administration, B.Z. and Y.L.; Funding Acquisition, B.Z. and Y.L. All authors have read and agreed to the published version of the manuscript.

Funding: This study was supported by the Scientific Production and Construction Corps (No.2021AB004), the National Natural Science Foundation of China (No. 31970020), the Research of the Degradation Mechanism of Macrofungi Metabolites on Wetland Water Pollutants (20190201256JC), and Key Projects of Jiangxi Province Key R\&D Plan (20212BBF61002).

Institutional Review Board Statement: Not applicable.

Informed Consent Statement: Not applicable.

Data Availability Statement: Data relevant to this research can be found here: https:/ /www.ncbi. nlm.nih.gov/; https:/ /www.mycobank.org/; https://www.treebase.org/treebase-web/home.html, accessed on 15 January 2022. 
Acknowledgments: We sincerely thank Zheng-Xiang Qi, Xin-Ya Yang, and Ya-Jie Liu of the Engineering Research Center of Edible and Medicinal Fungi, Ministry of Education, Jilin Agricultural University for their help in the experiment.

Conflicts of Interest: The authors declare no conflict of interest.

\section{References}

1. Chevallier, F.F. Flore Générale des Environs de Paris. Available online: https://bibdigital.rjb.csic.es/idurl/1/11834 (accessed on 20 December 2021).

2. Zhang, M. Molecular Phylogenetic Studies on the Family Boletaceae in Southern China, and Taxonomic Study on the Genus Aureoboletus in China. Ph.D. Thesis, South China University of Technology, Guangzhou, China, 2016.

3. Li, Y.; Li, T.H.; Yang, Z.L.; Bau, T.; Dai, Y.C. Atlas of Chinese Macrofungal Resources; Central Plains Farmers Press: Zhengzhou, China, 2016; pp. 1068-1148.

4. Roman, M.D.; Claveria, V.; Miguel, A.M. A revision of the descriptions of ectomycorrhizas published since 1961. Mycol. Res. 2005, 109, 1063-1104. [CrossRef] [PubMed]

5. Wu, G.; Li, Y.C.; Zhu, X.T.; Zhao, K.; Han, L.H.; Cui, Y.Y.; Li, F.; Xu, J.P.; Yang, Z.L. One hundred noteworthy boletes from China. Fungal Divers. 2016, 81, 25-188. [CrossRef]

6. Yang, Z.L.; Wu, G.; Li, Y.C.; Wang, X.H.; Cai, Q. Common Edible and Poisonous Mushrooms of Southwestern China; Science Press: Beijing, China, 2021.

7. Murrill, W.A. The Boletaceae of North America-I. Mycologia 1909, 1, 4-18. [CrossRef]

8. Smith, A.H.; Thiers, H.D. Boletes of Michigan; The University of Michigan Press: Ann Arbor, MI, USA, 1971.

9. Singer, R.; Williams, R. Some boletes from Florida. Mycologia 1992, 84, 724-728. [CrossRef]

10. Baroni, T.J. Boletus aurantiosplendens sp. nov. from the southern Appalachian Mountains with notes on Pulveroboletus auriflammeus, Pulveroboletus melleouluteus and Boletus auripes. Bull. Buffalo Soc. Nat. Sci. 1998, 36, 245-255.

11. Baroni, T.J.; Bessette, A.E.; Roody, W.C. Boletus patrioticus-A new species from the eastern United States. Bull. Buffalo Soc. Nat. Sci. 1998, 36, 265-268.

12. Farid, A.; Gelardi, M.; Angelini, C.; Franck, A.; Costanzo, F.; Kaminsky, L.; Ercole, E.; Baroni, T.; White, A.; Garey, J. Phylloporus and Phylloboletellus are no longer alone: Phylloporopsis gen. nov. (Boletaceae), a new smooth-spored lamellate genus to accommodate the American species Phylloporus Boletinoides. Fungal Syst. Evol. 2018, 2, 341. [CrossRef]

13. Vizzini, A.; Simonini, G.; Ercole, E.; Voyron, S. Boletus mendax, a new species of Boletus sect. Luridi from Italy and insights on the B. luridus complex. Mycol. Prog. 2014, 13, 95-109. [CrossRef]

14. Ortiz-Santana, B.; Roody, W.C.; Both, E.E. A new arenicolous Boletus from the Gulf Coast of northern Florida. Mycotaxon 2009, 107, 243-247. [CrossRef]

15. Ortiz-Santana, B.; Bessette, A.E.; McConnell, O.L. Boletus durhamensis sp. nov. from North Carolina. Mycotaxon 2016, 131, 703-715. [CrossRef]

16. Frank, J.; Siegel, N.; Schwarz, C.; Araki, B.; Vellinga, E. Xerocomellus (Boletaceae) in western North America. Fungal Syst. Evol. 2020, 6, 265. [CrossRef]

17. Crous, P.; Wingfield, M.; Lombard, L.; Roets, F.; Swart, W.; Alvarado, P.; Carnegie, A.; Moreno, G.; Luangsaard, J.; Thangavel, R. Fungal Planet description sheets: 951-1041. Persoonia 2019, 43, 223. [CrossRef]

18. Farid, A.; Franck, A.R.; Bolin, J.; Garey, J.R. Expansion of the genus Imleria in North America to include Imleria floridana, sp. nov., and Imleria pallida, comb. nov. Mycologia 2020, 112, 423-437. [CrossRef]

19. Farid, A.; Bessette, A.R.; Bolin, J.A.; Kudzma, L.V.; Franck, A.R.; Garey, J.R. Investigations in the boletes (Boletaceae) of southeastern USA: Four novel species and three novel combinations. Mycosphere 2021, 12, 1038-1076. [CrossRef]

20. Taylor, J.W.; Jacobson, D.J.; Kroken, S.; Kasuga, T.; Geiser, D.M.; Hibbett, D.S.; Fisher, M.C. Phylogenetic species recognition and species concepts in fungi. Fungal Genet. Biol. 2000, 31, 21-32. [CrossRef]

21. Nuhn, M.E.; Binder, M.; Taylor, A.F.; Halling, R.E.; Hibbett, D.S. Phylogenetic overview of the Boletineae. Fungal Biol. 2013, 117, 479-511. [CrossRef]

22. Wu, G.; Feng, B.; Xu, J.P.; Zhu, X.T.; Li, Y.C.; Zeng, N.K.; Hosen, M.I.; Yang, Z.L. Molecular phylogenetic analyses redefine seven major clades and reveal 22 new generic clades in the fungal family Boletaceae. Fungal Divers. 2014, 69, 93-115. [CrossRef]

23. Wilson, A.W.; Binder, M.; Hibbett, D.S. Diversity and evolution of ectomycorrhizal host associations in the Sclerodermatineae (Boletales, Basidiomycota). New Phytol. 2012, 194, 1079-1095. [CrossRef]

24. Wu, G.; Miyauchi, S.; Morin, E.; Kuo, A.; Drula, E.; Varga, T.; Kohler, A.; Feng, B.; Cao, Y.; Lipzen, A. Evolutionary innovations through gain and loss of genes in the ectomycorrhizal Boletales. New Phytol. 2022, 233, 1383-1400. [CrossRef]

25. Zhao, K.; Wu, G.; Yang, Z.L. A new genus, Rubroboletus, to accommodate Boletus sinicus and its allies. Phytotaxa 2014, 188, 61-77. [CrossRef]

26. Zhu, X.T.; Wu, G.; Zhao, K.; Halling, R.E.; Yang, Z.L. Hourangia, a new genus of Boletaceae to accommodate Xerocomus cheoi and its allied species. Mycol. Prog. 2015, 14, 1-10. [CrossRef]

27. Wu, G.; Zhao, K.; Li, Y.C.; Zeng, N.K.; Feng, B.; Halling, R.E.; Yang, Z.L. Four new genera of the fungal family Boletaceae. Fungal Divers. 2016, 81, 1-24. [CrossRef] 
28. Chai, H.; Liang, Z.Q.; Xue, R.; Jiang, S.; Luo, S.H.; Wang, Y.; Wu, L.L.; Tang, L.P.; Chen, Y.; Hong, D. New and noteworthy boletes from subtropical and tropical China. MycoKeys 2019, 46, 55. [CrossRef] [PubMed]

29. Li, M.X.; Wu, G.; Yang, Z.L. Four New Species of Hemileccinum (Xerocomoideae, Boletaceae) from Southwestern China. J. Fungi 2021, 7, 823. [CrossRef]

30. Gelardi, M.; Vizzini, A.; Ercole, E.; Horak, E.; Ming, Z.; Li, T.H. Circumscription and taxonomic arrangement of Nigroboletus roseonigrescens gen. et sp. nov., a new member of Boletaceae from tropical South-Eastern China. PLoS ONE 2015, 10, e0134295. [CrossRef]

31. Cui, Y.Y.; Feng, B.; Wu, G.; Xu, J.P.; Yang, Z.L. Porcini mushrooms (Boletus sect. Boletus) from China. Fungal Divers. 2016, 81, 189-212. [CrossRef]

32. Liang, Z.Q.; An, D.Y.; Juang, S.; Su, M.S.; Zeng, N.K. Butyriboletus hainanensis (Boletaceae, Boletales), a new species from tropical China. Phytotaxa 2016, 267, 256-262. [CrossRef]

33. Arora, D.; Frank, J.L. Clarifying the butter Boletes: A new genus, Butyriboletus, is established to accommodate Boletus sect. Appendiculati, and six new species are described. Mycologia 2014, 106, 464-480. [CrossRef]

34. Zeng, N.K.; Chai, H.; Jiang, S.; Xue, R.; Wang, Y.; Hong, D.; Liang, Z.Q. Retiboletus nigrogriseus and Tengioboletus fujianensis, two new boletes from the south of China. Phytotaxa 2018, 367, 45-54. [CrossRef]

35. Kornerup, A.; Wanscher, J.H. Methuen Handbook of Colour, 3rd ed.; Pavey, D., Ed.; Eyre Methuen: London, UK, 1978.

36. Imler, L. Recherches sur les bolets. Bull. Soc. Mycol. Fr. 1950, 66, 177-203.

37. Biketova, A.Y.; Gelardi, M.; Smith, M.E.; Simonini, G.; Healy, R.A.; Taneyama, Y.; Vasquez, G.; Kovács, A.; Nagy, L.G.; Wasser, S.P.; et al. Reappraisal of the Genus Exsudoporus (Boletaceae) Worldwide Based on Multi-Gene Phylogeny, Morphology and Biogeography, and Insights on Amoenoboletus. J. Fungi 2022, 8, 101. [CrossRef]

38. Zhu, X.T.; Li, Y.C.; Wu, G.; Feng, B.; Zhao, K.; Gelardi, M.; Kost, G.W.; Yang, Z.L. The genus Imleria (Boletaceae) in East Asia. Phytotaxa 2014, 191, 81-98. [CrossRef]

39. White, T.J.; Bruns, T.; Lee, S.; Taylor, J. Amplification and direct sequencing of fungal ribosomal RNA genes for phylogenetics. PCR Protoc. Guide Methods Appl. 1990, 18, 315-322.

40. Cubeta, M.; Echandi, E.; Abernethy, T.; Vilgalys, R. Characterization of anastomosis groups of binucleate Rhizoctonia species using restriction analysis of an amplified ribosomal RNA gene. Phytopathology 1991, 81, 1395-1400. [CrossRef]

41. Vilgalys, R.; Hester, M. Rapid genetic identification and mapping of enzymatically amplified ribosomal DNA from several Cryptococcus species. J. Bacteriol. 1990, 172, 4238-4246. [CrossRef]

42. Rehner, S.A.; Buckley, E. A Beauveria phylogeny inferred from nuclear ITS and EF1- $\alpha$ sequences: Evidence for cryptic diversification and links to Cordyceps teleomorphs. Mycologia 2005, 97, 84-98. [CrossRef]

43. Zhang, M.; Li, T.H.; Song, B. Two new species of Chalciporus (Boletaceae) from southern China revealed by morphological characters and molecular data. Phytotaxa 2017, 327, 47-56. [CrossRef]

44. Kuo, M.; Ortiz-Santana, B. Revision of leccinoid fungi, with emphasis on North American taxa, based on molecular and morphological data. Mycologia 2020, 112, 197-211. [CrossRef]

45. Hall, T. BioEdit: A user-friendly biological sequence alignment editor and analysis program for Windows 95/98/NT. Nucleic Acids Symp. Ser. 1999, 41, 95-98. [CrossRef]

46. Lanfear, R.; Frandsen, P.B.; Wright, A.M.; Senfeld, T.; Calcott, B. PartitionFinder 2: New methods for selecting partitioned models of evolution for molecular and morphological phylogenetic analyses. Mol. Biol. Evol. 2017, 34, 772-773. [CrossRef]

47. Kalyaanamoorthy, S.; Minh, B.Q.; Wong, T.K.; Von Haeseler, A.; Jermiin, L.S. ModelFinder: Fast model selection for accurate phylogenetic estimates. Nat. Methods 2017, 14, 587-589. [CrossRef]

48. Nguyen, L.T.; Schmidt, H.A.; Von Haeseler, A.; Minh, B.Q. IQ-TREE: A fast and effective stochastic algorithm for estimating maximum-likelihood phylogenies. Mol. Biol. Evol. 2015, 32, 268-274. [CrossRef]

49. Ronquist, F.; Teslenko, M.; Van Der Mark, P.; Ayres, D.L.; Darling, A.; Höhna, S.; Larget, B.; Liu, L.; Suchard, M.A.; Huelsenbeck, J.P. MrBayes 3.2: Efficient Bayesian phylogenetic inference and model choice across a large model space. Syst. Biol. 2012, 61, 539-542. [CrossRef]

50. Binder, M.; Hibbett, D.S. Molecular systematics and biological diversification of Boletales. Mycologia 2006, 98, 971-981. [CrossRef]

51. Feng, B.; Xu, J.P.; Wu, G.; Zeng, N.K.; Li, Y.C.; Tolgor, B.; Kost, G.W.; Yang, Z.L. DNA sequence analyses reveal abundant diversity, endemism and evidence for Asian origin of the porcini mushrooms. PLoS ONE 2012, 7, e37567. [CrossRef]

52. Binder, M.; Bresinsky, A. Retiboletus, a new genus for a species-complex in the Boletaceae producing retipolides. Feddes Repert. Z. Bot. Taxon. Geobot. 2002, 113, 30-40. [CrossRef]

53. Zhao, K.; Wu, G.; Halling, R.E.; Yang, Z.L. Three new combinations of Butyriboletus (Boletaceae). Phytotaxa $2015,234,51-62$. [CrossRef]

54. Zhao, K.; Wu, G.; Feng, B.; Yang, Z.L. Molecular phylogeny of Caloboletus (Boletaceae) and a new species in East Asia. Mycol. Prog. 2014, 13, 1127-1136. [CrossRef]

55. Halling, R.E.; Nuhn, M.; Fechner, N.A.; Osmundson, T.W.; Soytong, K.; Arora, D.; Hibbett, D.S.; Binder, M. Sutorius: A new genus for Boletus eximius. Mycologia 2012, 104, 951-961. [CrossRef]

56. Šutara, J.; Janda, V.; Kř́ž, M.; Graca, M.; Kolařík, M. Contribution to the study of genus Boletus, section Appendiculati: Boletus roseogriseus sp. nov. and neotypification of Boletus fuscoroseus Smotl. Czech Mycol. 2014, 66, 1-37. [CrossRef]

57. Li, Y.C.; Ortiz-Santana, B.; Zeng, N.K.; Feng, B.; Yang, Z.L. Molecular phylogeny and taxonomy of the genus Veloporphyrellus. Mycologia 2014, 106, 291-306. [CrossRef] [PubMed] 
58. Li, Y.C.; Feng, B.; Yang, Z.L. Zangia, a new genus of Boletaceae supported by molecular and morphological evidence. Fungal Divers. 2011, 49, 125-143. [CrossRef]

59. Krpata, D.; Peintner, U.; Langer, I.; Fitz, W.J.; Schweiger, P. Ectomycorrhizal communities associated with Populus tremula growing on a heavy metal contaminated site. Mycol. Res. 2008, 112, 1069-1079. [CrossRef] [PubMed]

60. Osmundson, T.W.; Robert, V.A.; Schoch, C.L.; Baker, L.J.; Smith, A.; Robich, G.; Mizzan, L.; Garbelotto, M.M. Filling gaps in biodiversity knowledge for macrofungi: Contributions and assessment of an herbarium collection DNA barcode sequencing project. PLoS ONE 2013, 8, e62419. [CrossRef]

61. Iotti, M.; Barbieri, E.; Stocchi, V.; Zambonelli, A. Morphological and molecular characterisation of mycelia of ectomycorrhizal fungi in pure culture. Fungal Divers. 2005, 19, 51-68.

62. Nygren, C.M.; Edqvist, J.; Elfstrand, M.; Heller, G.; Taylor, A.F. Detection of extracellular protease activity in different species and genera of ectomycorrhizal fungi. Mycorrhiza 2007, 17, 241-248. [CrossRef]

63. Martin, M.P.; Raidl, S. The taxonomic position of Rhizopogon melanogastroides (Boletales). Mycotaxon 2002, 84, 221-228.

64. Smith, M.E.; Douhan, G.W.; Rizzo, D.M. Ectomycorrhizal community structure in a xeric Quercus woodland based on rDNA sequence analysis of sporocarps and pooled roots. New Phytol. 2007, 174, 847-863. [CrossRef]

65. Mello, A.; Ghignone, S.; Vizzini, A.; Sechi, C.; Ruiu, P.; Bonfante, P. ITS primers for the identification of marketable boletes. J. Biotechnol. 2006, 121, 318-329. [CrossRef]

66. Palmer, J.M.; Lindner, D.L.; Volk, T.J. Ectomycorrhizal characterization of an American chestnut (Castanea dentata)-dominated community in Western Wisconsin. Mycorrhiza 2008, 19, 27-36. [CrossRef]

67. Li, H.; Wei, H.; Peng, H.; Ding, H.; Wang, L.; He, L.; Fu, L. Boletus roseoflavus, a new species of Boletus in section Appendiculati from China. Mycol. Prog. 2014, 13, 21-31. [CrossRef]

68. Takahashi, H.; Taneyama, Y.; Degawa, Y. Notes on the boletes of Japan 1. Four new species of the genus Boletus from central Honshu, Japan. Mycoscience 2013, 54, 458-468. [CrossRef]

69. Janda, V.; Kř́žž, M.; Kolařík, M. Butyriboletus regius and Butyriboletus fechtneri: Typification of two well-known species. Czech Mycol. 2019, 71, 1-32. [CrossRef]

70. Thiers, H.D. California Mushrooms—A Field Guide to the Boletes; Hafner Press: New York, NY, USA, $1975 ;$ p. 261.

71. Muñoz, J.A. Boletus s.l. (excl. Xerocomus). In Fungi Europaei 2; Candusso Editrice: Bardolino, Italy, 2005 ; pp. 428-432.

72. Heykoop, M. Morphology and taxonomy of Boletus queletii var. discolor, a rare bolete resembling Boletus erythropus. Mycotaxon $1995,56,115-123$

73. Marsico, O.; Musumeci, E. Boletus adalgisae sp. nov. Boll. Assoc. Micol. Ecol. Romana 2011, 27, 3-15.

74. Seaver, F.J.; John, N.C.; Murrill, W.A.; George, L.Z.; Fred, W.; Singer, R. Notes and Brief Articles. Mycologia 1945, 37, 792-799. [CrossRef]

75. Pilát, A. Boletus gabretae sp. nov. bohemica ex affinitate Boleti junguillei (Quél.) Boud. Czech Mycol. 1968, 22, 167-170.

76. Murill, W.A. More Florida fungi. Lloydia 1946, 8, 263-290.

77. New York State Museum. Bulletin of the New York State Museum; University of the State of New York: New York, NY, USA, 1889; Volume 2, pp. 142-143.

78. De Queiroz, K. Species concepts and species delimitation. Syst. Biol. 2007, 56, 879-886. [CrossRef]

79. Truong, C.; Mujic, A.B.; Healy, R.; Kuhar, F.; Furci, G.; Torres, D.; Niskanen, T.; Sandoval-Leiva, P.A.; Fernández, N.; Escobar, J.M. How to know the fungi: Combining field inventories and DNA-barcoding to document fungal diversity. New Phytol. 2017, 214, 913-919. [CrossRef]

80. Bozok, F.; Assyov, B.; Taşkin, H. First records of Exsudoporus permagnificus and Pulchroboletus roseoalbidus (Boletales) in association with non-native Fagaceae, with taxonomic remarks. Phytol. Balc. 2019, 25, 13-27.

81. Liu, H.Y. Taxonomy and Resource Evaluation of Boletes in Northeastern China. Master's Thesis, Jilin Agricultural University, Changchun, China, 2020.

82. Muñoz, J.; Boletus, S.L. (Excl. Xerocomus): Strobilomycetaceae, Gyroporaceae, Gyrodontaceae, Suillaceae, Boletaceae. In Fungi Europaei 2; Edizioni Candusso: Alassio, Italy, 2005

83. Han, L.H.; Wu, G.; Horak, E.; Halling, R.; Xu, J.P.; Ndolo, E.; Sato, H.; Fechner, N.; Sharma, Y.; Yang, Z.L. Phylogeny and species delimitation of Strobilomyces (Boletaceae), with an emphasis on the Asian species. Persoonia 2020, 44, 113-139. [CrossRef] [PubMed]

84. Klofac, W. Schlüssel zur Bestimmung von Frischfunden der europäischen Arten der Boletales mit röhrigem Hymenophor. Osterr. Z. Pilzkd. 2007, 16, 187-279.

85. Vesterholt, J. Funga Nordica, Agaricoid, Boletoid, Cyphelloid and Gasteroid Genera; Nordsvamp: Copenhagen, Denmark, 2012; p. 9811021.

86. Gelardi, M. Contribution to the knowledge of Chinese boletes. II: Aureoboletus thibetanus sl, Neoboletus brunneissimus, Pulveroboletus macrosporus and Retiboletus kauffmanii (Part I). Riv. Micol. Romana 2017, 102, 13-30. 